\title{
Probabilistic transition of condition: render facades
}

\begin{tabular}{|c|c|}
\hline Journal: & Building Research \& Information \\
\hline Manuscript ID: & 13BR0015-RE.R3 \\
\hline Manuscript Type: & Research Paper \\
\hline Keywords: & durability, facades, building management, building envelope \\
\hline Other keywords: & $\begin{array}{l}\text { service life prediction, render facades, environmental conditions, Markov } \\
\text { chains }\end{array}$ \\
\hline Abstract: & $\begin{array}{l}\text { The service life of cement-rendered facades is closely related to the } \\
\text { environmental conditions that they are exposed to. The probability } \\
\text { distribution is determined for the degradation condition of render facades } \\
\text { considering different environmental exposures. A sample of } 100 \text { render } \\
\text { facades was subjected to meticulous fieldwork to determine their condition. } \\
\text { The analysis focuses on the environmental factors that most influence the } \\
\text { overall degradation of the facades, evaluated through the condition level. } \\
\text { Probabilistic models based on Markov chains are developed to predict the } \\
\text { evolution of facade deterioration according to exposure to outdoor } \\
\text { environmental conditions. The proposed model provides data on the } \\
\text { synergy between the degradation agents and the degradation condition of } \\
\text { render facades, the average time of permanence in each degradation level } \\
\text { and indications of the effect of degradation on the durability of render that } \\
\text { may be applied in the implementation and fine tuning of maintenance } \\
\text { procedures. Better understanding the durability of render facades allows a } \\
\text { more rational management of their maintenance, contributing to a } \\
\text { reduction of their life cycle costs. The proposed stochastic model provides } \\
\text { information that can be applied in the context of insurance policies, } \\
\text { allowing an evaluation of the risk of failure of coatings. }\end{array}$ \\
\hline
\end{tabular}

\section{SCHOLARONE}

Manuscripts 


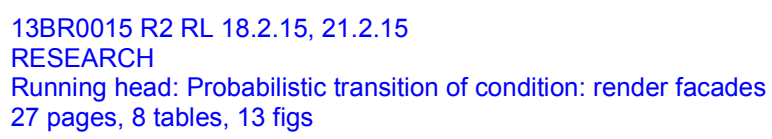

\title{
Probabilistic transition of condition: render facades
}

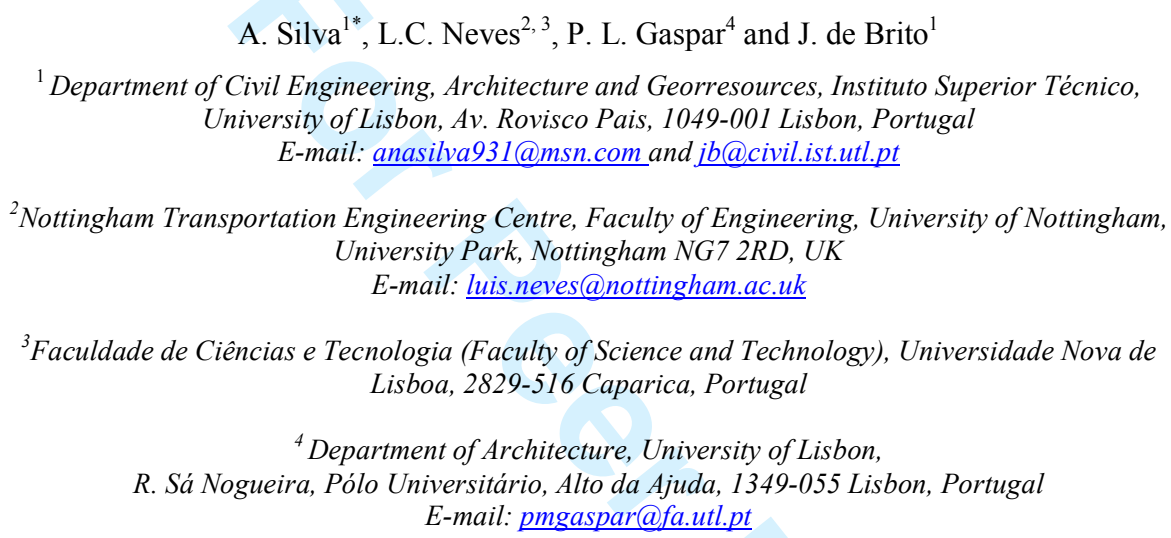

\begin{abstract}
The service life of cement-rendered facades is closely related to the environmental conditions that they are exposed to. The probability distribution is determined for the degradation condition of render facades considering different environmental exposures. A sample of 100 render facades was subjected to meticulous fieldwork to determine their condition. The analysis focuses on the environmental factors that most influence the overall degradation of the facades, evaluated through the condition level. Probabilistic models based on Markov chains are developed to predict the evolution of facade deterioration according to exposure to outdoor environmental conditions. The proposed model provides data on the synergy between the degradation agents and the degradation condition of render facades, the average time of permanence in each degradation level and indications of the effect of degradation on the durability of render that may be applied in the implementation and fine tuning of maintenance procedures. Better understanding the durability of render facades allows a more rational management of their maintenance, contributing to a reduction of their life cycle costs. The proposed stochastic model provides information that can be applied in the context of insurance policies, allowing an evaluation of the risk of failure of coatings.
\end{abstract}

Keywords: durability, service life prediction; render; facades; environmental conditions; Markov chains. 


\section{Introduction}

The increasing scarcity of funding for the maintenance and rehabilitation of infrastructures requires a more rational approach to decision making, in terms of inspection, maintenance and rehabilitation (Paulo et al., 2013). To enable this rational approach to maintenance, an efficient evaluation of the service life must be considered, which accounts for the material properties, the environmental conditions of exposure, the workmanship, the service conditions and the maintenance planning (ISO 15686-1:2000). However, few infrastructures are currently studied in a consistent and rational manner over their entire lifecycle, and decisions are made based mostly on subjective and programmatic criteria (Aikivuori, 1999).

The environmental conditions that affect buildings are dynamic and undergo changes due to anthropogenic and natural activities, which influences their impact on building elements (Costa et al., 2009). The interactions of cementitious material with the environment may jeopardize their durability and service life (Zivica et al., 2012). Given the variability and complexity degradation phenomena (Silva et al., 2012), service life analysis cannot be seen as an exact science (BSI 7543, 1992). In fact, the studies related to life-cycle analysis, condition prediction, and risk assessment involve a large number of uncertainties (Ellingwood, 2005). These uncertainties are due to two main reasons: the limited ability to predict future events and the intrinsic randomness of natural phenomena (e.g. environmental agents) (Bocchini et al., 2013). To overcome the uncertainty associated with current and future performance of a structure or of any building element, it is necessary to follow a probabilistic approach to service life prediction (Frangopol et al., 2004). Usually, this approach tends to be relatively complex, relying on the assumption that deterioration is a process described by random variables to such an extent that one cannot fully predict the next degradation stage - also referred to as 'condition' - based on the knowledge of the current degradation state of a building element (Moser, 2003). Therefore, the assessment of the life-cycle performance of a structure or building should be based on probabilistic analysis tools able to model the deterioration process over time (Basso et al., 2013). 


\begin{abstract}
This study sets out to evaluate the degradation of external render in a probabilistic framework, based on the visual inspections of the characteristics and condition of 100 facades inspected in Portugal, using Markov chains. Markov chains have been used to statistically represent the degradation phenomena of a manifold of infrastructures and infrastructure components (Handel, 2008). This stochastic method makes it possible to predict the probabilistic condition over time and to understand, in some detail, how the environmental exposure conditions contribute to the overall degradation of these facades, based on limited historical data. The results obtained provide stakeholders with information on the influence of exposure conditions on the degradation of render facades and thus become a tool for the development and implementation of maintenance strategies adapted to specific environmental contexts.
\end{abstract}

\title{
2. Background
}

Claddings can be described as the skin of the structure or a wall, which influence its safety and aesthetics (Flores and de Brito, 2010). As such, they act as the first layer of protection against environmental agents. Although these elements generally have a shorter service life than the structure, they must retain their characteristics as long as possible throughout the service life of the building and have the potential to be easily repaired or replaced, in order to comply with minimum performance levels (Silva et al., 2011). Render over a substrate (typically: masonry) is the most common type of cladding in Portugal, representing $62 \%$ of existing solutions (Flores-Colen et al., 2009; INE, 2001). The predominance of render is, essentially, a result of its low cost and the relative lack of expertise required for its execution, compared to other claddings and coatings (Gaspar and de Brito, 2005). However, the low investment in this type of cladding often implies unacceptable degradation levels of the facades, due to execution defects or poor maintenance (Freitas et al., 1999).

Three main groups of defects for modern render can be identified, with different levels of severity (Gaspar, 2009; Silva et al., 2013): (a) staining; (b) cracking; and (c) loss of adherence. Even though staining is often associated with moisture and damp (Chew and Ping, 2003), these defects have a relatively low impact on the functionality or durability of renders (Gaspar and de 


\section{Brito, 2008a). Cracking is a more serious defect than staining. However, by itself, it does not} jeopardise the safety or the normal use of a construction and it may even be a natural consequence of the behaviour of the various materials/components of the render (Bonshor and Bonshor, 2001). Nevertheless, cracking allows water penetration into the cladding and, sometimes, into the wall or structure, which in turn may lead to salt deposit, freeze-thaw cycles, detachment from the previous render layer or from the wall - or indeed a combination of different degradation mechanisms and defects - that may eventually compromise the functionality and the durability of renders (Hansen et al., 1999). Finally, loss of adherence is the most serious type of defect. It usually occurs as a consequence of different factors, including the combination of cracking and water penetration as referred to above, leading quite often to spalling and detachment and to the end of the cladding's service life.

The degradation phenomena affecting construction can be defined as a transition process through different condition states (Garavaglia et al., 2004). There are several methods for assessing the degradation of render facades. A visual inspection usually suffices, since parameters like "type of defects", "condition of defects" and "extent of defects" can be assessed (Straub, 2003; Rodrigues et al., 2013). In this study, the model proposed is based on a visual survey of the degradation state of 100 current external renders analysed in Portugal. Case studies were randomly selected and assessed provided there was reliable information on the maintenance history of each case, because age (i.e. the length of time since the last overall maintenance action) was the critical variable of the sample. The data on façade degradation collected during field work are combined in an engineering model to define the overall façade condition, from which it is possible to assess the facades' durability. Additional information is also collected (location, architectural working drawings, documentation from Town Halls, and other relevant data) to better understand each case study. This method does not usually require costly equipment, and is often sufficient to determine the degradation state of the elements under inspection (Silva et al., 2011). Nevertheless, visual inspections have some limitations since their accuracy depends significantly on the experience/background and classification 
criteria of the surveyor. Also, this method also depends on the atmospheric conditions at the time of the inspection (e.g. the difficulty in detecting anomalies in smooth and dark claddings in direct sunlight). Finally, it is not possible to assess hidden defects that may jeopardize the element under inspection but may be invisible to the surveyor (such as a detachment from the wall, with no other alteration to the surface of the render). To quantify the overall deterioration condition of a facade, various authors propose qualitative and quantitative deterioration levels, ranging from the best possible condition to the highest degradation level, and a number of different methods (Shohet and Paciuk, 2004, 2006; Moser, 2004). In this study, the defects associated to render facades were grouped into five categories, ranging from A (no visually detectable degradation - most favourable situation) to E (highest degradation level), as shown in Table 1. Level A (more favourable situation) corresponds to a mortar with no visual deterioration and no detached elements. Level B corresponds to render with good performance, minor staining in the surface and eventual presence of microorganisms, no signs of detachment and the odd presence of small cracking in localized areas. Level C corresponds to minor degradation, consisting of soot and dirt deposition in the surface of the façade, with the possibility of localized minor detachments or perforations of the mortar and presence of dark patches of damp and dirt, often associated with microorganisms and algae. Level D corresponds to renders with moderate degradation, incomplete mortar surface due to spalling and detachment of mortar patches, wide or extensive cracking and the possibility of dark patches with probable presence of fungi or algae. Level E corresponds to extensive degradation of the render (worst case scenario), with incomplete mortar surface due to detachment and falling off of mortar patches, wide or extensive cracking and heavy staining with presence of algae and/or fungi, associated to permanent presence of moisture. Each degradation condition is associated with a qualitative scale - based on the evaluation of the physical and visual condition of the sample analysed - and a quantitative index that depicts the global performance of the facades - the severity index.

The degradation severity index of a facade is a numeric indicator that expresses the overall 
condition of a facade and allows a comparison between different case studies, with distinct characteristics and exposure conditions, and thus enables the use of statistical tools from field data as in our research (Gaspar, 2009). This degradation index is obtained as the ratio between the extent of the façade degradation -weighted according to the degradation level and the severity of defects - and a reference area, equivalent to the maximum theoretical extent of the degradation for the facade in question (Gaspar and de Brito, 2008b; 2011):

$S=\frac{\Sigma\left(A_{n} \times k_{n} \times k_{a, n}\right)}{A \times k}$

where $\mathrm{S}$ is the weighted severity of degradation of the facade $(\%) ; \mathrm{A}_{\mathrm{n}}$ is the area of coating affected by a defect $n ; k_{n}$ is the multiplying factor for defect $n$, as a function of its condition (between 0 and 4); $\mathrm{k}_{\mathrm{a}, \mathrm{n}}$ is the weighting coefficient corresponding to the relative importance of each defect $\left(\mathrm{k}_{\mathrm{a}, \mathrm{n}} \in \mathrm{R}^{+}\right)$based on the cost of repair of defects; $\mathrm{k}$ is the weighting factor equal to the highest degradation level in the façade (4, in the case of render); A is the total area of the cladding. Equation (1) allows the quantification of the overall degradation of the facade, which is then classified according to its overall condition level and related to the qualitative assessment of the facade, from A to E, as shown in Table 1. In terms of durability, it was considered that level D or S $>20 \%$ represents the minimum accepted level of performance and thus stands for the service life limit of the renders studied (Gaspar and de Brito, 2005), despite the fact that different stakeholders may have distinct minimum reference levels resulting from available funding, type of use or perception of risk, to name but a few aspects.

\section{Markov chains}

Within the methods to evaluate stochastically the future condition of the building elements, Markov chains are a special case of Markov processes whose development can be treated as a series of transitions between given states (Morcous and Lounis, 2005). Markov chains can be used to emulate the evolution of the degradation state of constructions, defining the probability of a future state based only on the present condition, independently of previous deterioration 


\begin{abstract}
history (Parzen, 1962; Neves et al., 2006). This property of Markov chains is usually called
\end{abstract} "loss of memory" (Durango-Cohen and Madanat, 2006).

Concerning the time of transition between states of deterioration, Markov chains can be divided in two common types: discrete and continuous. Discrete chains are useful when transitions can only occur at specific instants. Continuous chains are more appropriate when transitions can occur at any time, as is, in general, the case of deteriorating performance. The uncertainty in the rate of transitions between the states is defined by a transition probability matrix (denoted $\mathrm{P}$ ) for discrete-time processes and by an intensity matrix (denoted Q) for continuous-time processes (Kallen, 2009). In this study, only the continuous-time models are analysed.

Augenbroe and Park (2002) argue that Markov chain models can describe the randomness associated to building system performance and can therefore be used for systematic decisions regarding replacement of building components. In fact, Markov chains have been successfully applied to various fields of civil engineering, particularly in predicting future condition of bridges (Thompson et al., 1998; Hawk and Small, 1998; Morcous et al., 2003; Robelin and Madanat, 2007; Bocchini et al., 2013), optimization of maintenance actions (Van Winden and Dekker, 1998; Lounis and Vanier, 2000), life-cycle assessment analysis (Ortiz- García et al., 2006) and service life prediction of constructions elements (Mc Duling, 2006). When used to model deterioration processes, it is assumed that, in a given infinitesimal time period, transitions can only occur between one state and the next one. It is also assumed that improvement from one given condition cannot occur (i.e. degradation reversion is impossible), and every observed improvement corresponds to an inspection error or undocumented maintenance action. Under these assumptions, the elements in the intensity matrix Q must respect:

$\left\{\begin{array}{cc}q_{i i}=-q_{i j} \quad \text { for } j=i+1 \\ q_{i j}=0 \text { for } j \neq i \text { and } j \neq i+1\end{array}\right.$

In equation (3) a generic intensity matrix Q is show (Singer, 1981). 
The transition between states of degradation depends solely on the last recorded state and the transition rate between the current state and future state (given by the matrix Q). Based on the transition rates, the probability of transition between states of condition and the mean probability of permanence in these states can be computed using Chapman-Kolmogorov differential equation (Norris, 1997):

$\frac{d}{d t} P(\Delta t)=Q \cdot P(\Delta t)$

The solution of this system of differential equations is given by (Carrington et al., 2005):

$P(\Delta t)=\exp (Q \cdot \Delta t)=\sum_{n=0}^{\infty} \frac{\Delta t^{n} \cdot Q^{n}}{n !}$

where $\Delta t$ represents the time interval considered.

Thus, it is possible to relate the infinitesimal generator matrix $Q$ with the Markovian transition matrix $P$ (Cox and Miller, 1965; Bladty and Sorensen, 2009).

The estimation of the intensity matrix $Q$ can be made by various methods. Optimization procedures are generally regarded as a consistent and accurate methodology to estimate the intensity matrix $Q$, ensuring the efficiency of the degradation model. The optimization of matrix $Q$ is based on the concept of maximum likelihood described by Lawless (1982) and Kalbfleisch and Lawless (1985). The likelihood is defined as the predicted probability of occurrence of the observed transitions:

$L(Q)=\prod_{i=1}^{m} \prod_{i, j=1}^{k} P_{i j}$ 
where $i$ is the condition state in the initial instant, $j$ is the condition state in the final instant, $m$ is the number of elements, $k$ is the number of intervals between inspections and $P_{i j}$ is the probability of transition from the condition $i$ to condition $j$.

Currently, a great variety of highly efficient optimization tools are available in commercial software. In this study, the optimization of the infinitesimal generator matrix $Q$ is carried out using the function fmincon (constrained nonlinear optimization) available in MATLAB (Higham and Higham, 2005).

\subsection{Probabilistic analysis of degradation of render over time}

As shown in Table 1, the degradation condition of render facades is characterized and classified according to a range of discrete variables from A (no visible degradation) to $\mathrm{E}$ (widespread deterioration, requiring an immediate corrective action). In this study, only the initial condition (assuming that at time zero the render is in condition A) and final condition, corresponding to the inspection date, are known. Having defined the scale of degradation of render facades, it is possible to establish a degradation model using Markov chains. With the function fmincon, one proceeds to optimize the intensity matrix $(Q)$, resulting in:

$Q=\left[\begin{array}{l}q_{0,1} \\ q_{1,2} \\ q_{2,3} \\ q_{3,4}\end{array}\right]=\left[\begin{array}{l}0,4016 \\ 0,2819 \\ 0,0994 \\ 0,0761\end{array}\right]$

The data in Table 2 allow an evaluation of the efficiency of the Markov chains model. This table shows the number of predicted and observed coatings in each state of degradation. The mean relative error obtained for the estimated number of cases belonging to each degradation condition is relatively low: the mean relative error for all states is $7.55 \%$ and all results are lower than $16 \%$. Taking into account all the variability associated with the degradation phenomena it is considered that the model is suitable and able to correctly classify the cases analysed.

After the estimation of the intensity matrix $(Q)$, it is possible to determine the mean time of 
permanence in each degradation state, $T_{i}$ (Figure 1):

$T_{i}=\frac{1}{q_{i j}}$

The results show that transitions between condition states of facades occur faster for less deteriorated facades (changes from level A to level B occur in only 2.5 years). Renders remain for a longer period of time in higher degradation levels (conditions $\mathrm{C}$ and D). The transition between levels associated with higher degradation states imply the presence of a larger number of defects and/or more hazardous defects, including simultaneous occurrence of defects and synergies between them.

Figure 2 and Table 3 illustrate the probabilistic distribution of the degradation condition over time, based on the Markov chain presented in equation (7). By default, transition times are exponentially distributed, for it is assumed that the probability of transition between states is constant in time. This is considered an acceptable simplification of a complex reality given the relatively small sample of available data, despite the fact that renders with different ages may have distinct probabilities of transition between states. (However, this concern is beyond the scope of this paper). As expected, the probability of renders being in level A decreases rapidly over time, being lower than $2 \%$ at year 10 . The probability of renders being in level B initially increases and reaches a peak at around year 3 (probability of $43.5 \%$ ) and then steadily decreases as the probability for higher levels of degradation increases. Probability for levels C and D display skewed distribution curves, with rapid increases and peaks at year $10(\mathrm{P}=51.4 \%)$ and year $19(\mathrm{P}=38.5 \%)$ for levels $\mathrm{C}$ and $\mathrm{D}$ respectively. Finally, the probability of level E steadily increases with age as expected, i.e. it is practically nil before year 9 , and above $75 \%$ after year 38.

\subsection{Probabilistic analysis of degradation relative to the exposure conditions}

For any given building or structural component, there is a distinct anticipated service life which is directly dependent on its environmental conditions (Cheung and Kyle, 1996). Facade degradation occurs due to the combined influence of degradation agents such as rain, wind, 
sunlight, biological agents, and atmospheric pollutants (Parnham, 1997; Atkinson and Snape, 1999; Chew and Tan, 2003). Render facades show significant differences in terms of deterioration as a result of the great variability of their exposure to environmental conditions. In this study, the degradation factors considered are: distance from the sea, exposure to dampness, exposure to the combined action to rain and wind, and distance from pollutant sources.

\subsubsection{Maritime influence}

Maritime environments are particularly damaging to external renders. Wind containing sea spray causes the progressive deposition of salts on the external surfaces of the facades (Hossain et al., 2009). These salts can penetrate the mortar, by ionic diffusion, and crystallize inside the material, causing the degradation of its physical structure (Sabbioni et al., 2001), affecting its durability and reducing the service life of render (Soroka and Carmel, 1987). Figure 3 shows the mean time of permanence in each degradation state according to distance from the sea. Figure 4 shows the evolution in time of the probability of the coatings belonging to each degradation state. Facades located more than $3.5 \mathrm{~km}$ from the sea remain longer in level A, with a probability of $50 \%$ of being in level A at year 5. Facades located along the coast (less than $3.5 \mathrm{~km}$ from the sea) are more prone to higher degradation levels: such cases change from level A to level B in less than 1 year, and after year 5 the probability of being in level A is practically nil. For facades located on the coast, the probability of being in level B peaks at around year $1(\mathrm{P}=55.7 \%)$, the probability of being in level $\mathrm{C}$ peaks at around year $6(\mathrm{P}=58 \%)$ and the probability of being in level $\mathrm{D}$ reaches a peak $(\mathrm{P}=47 \%)$ at around year 16 . For facades located off the coast (more than $3.5 \mathrm{~km}$ from the sea), these levels are reached significantly later: year 4 for level B (probability of $16.5 \%$ ), year 14 for level $\mathrm{C}(\mathrm{P}=52 \%)$ and year 16 for level $\mathrm{E}$ (probability of 5\%).

\subsubsection{Exposure to damp}

Damp is the main source of defects in buildings worldwide (Lourenço et al., 2006). Dampness associated with salt leaching can cause aesthetic degradation and/or structural damage to exterior building facades (Rirsch et al., 2011). The presence of moisture in building's facade can 
originate the presence of cracking, staining and soiling of the surface of the render, biological growth (presence of algae and other microorganisms), among other defects. Exposure to damp was classified according to a combination of average annual rainfall and relative humidity (RH). Average annual rainfall is relatively low for the cases studied, ranging from $400 \mathrm{~mm}$ to $600 \mathrm{~mm}$. Relative humidity is relatively high since all buildings are located less than $20 \mathrm{~km}$ from the shore. Thus, a distinction was made between cases located in areas with relative humidity of $70 \%$ to $75 \%$ and those exposed to values of $75 \%$ to $80 \%$, for which the occurrence of corrosion phenomena strongly increases. Three categories were adopted: i) favourable, for situations of buildings located in areas with $400 \mathrm{~mm}$ to $500 \mathrm{~mm}$ of annual rainfall and $\mathrm{RH}$ under $75 \%$, without the influence of prevailing winds from sea or rivers; ii) moderate, for facades located in areas with annual rainfall of $500 \mathrm{~mm}$ to $600 \mathrm{~mm}$ and $\mathrm{RH}$ under $75 \%$; and iii) unfavourable conditions for renders located in areas with annual rainfall higher than $500 \mathrm{~mm}$ and $\mathrm{RH}$ higher than $75 \%$ or under direct influence of prevailing winds from the sea or from a river front. It should be noted that exposure to damp provides an indication of an expected probability of occurrence of defects, but does not specifically determine which type of degradation mechanism may occur. Defects related to damp and moisture can have a wide range of manifestations, from runoff staining, ascending damp, biological growth, etc. These defects have been independently assessed for each case studied and generically grouped under staining defects.

Figures 5 and 6 show the mean time of permanence in a degradation state and the probability of belonging to a degradation state respectively, according to exposure to damp. Facades with low levels of exposure to damp remain for a longer period in level A, having a maximum probability of belonging to degradation level B of $42 \%$ at year 4 while, under a severe exposure, this peak occurs at year 1 approximately, with $\mathrm{P}=35.6 \%$, and for moderate exposure it occurs at year 3 approximately, with $\mathrm{P}=49.8 \%$. Concerning level $\mathrm{C}$, facades with low damp exposure have a maximum probability of belonging to this degradation level $(\mathrm{P}=57.2 \%)$ after year 10 while for a moderate exposure this peak is reached at year $8(\mathrm{P}=34.3 \%)$, and for severe exposure at year 3 approximately $(\mathrm{P}=76.9 \%)$. A similar trend occurs for level $\mathrm{D}$. 


\subsubsection{Exposure to wind-driven rain}

The combined action of wind and rain changes the trajectory of raindrops, the way they impact the surfaces of the facade and the pattern of runoff flow along the facade (Choi, 1999; Gaspar and de Brito, 2008b). In this study, the criteria adopted in the quantification of driven-rain incidence are associated to the level of protection of the façade, since there is no reliable available weather data at a local scale for the sample studied. The level of protection takes into consideration the height of the building and the density of ground occupation in the surrounding area, according to the parameters defined by Gaspar (2009): i) good protection level - for low-rise buildings (up to 2 storeys high), in densely populated areas, protected from prevailing winds by other buildings, adjacent hills or vegetation; ii) current protection level - for medium-high buildings, in populated urban areas, protected from prevailing winds by other buildings, adjacent hills or vegetation; iii) poor protection level - for buildings with more than 4-storeys or in open country or crossroads. Figures 7 and 8 show the mean time of permanence in each degradation state of the facades as a function of the exposure level to wind-driven rain and the probabilistic distribution of the degradation condition as a function of the age of the render and the exposure level of the facades, respectively. Render with current protection level (moderate exposure to wind-rain action) are the ones that remain longer in degradation level A (i.e., with no apparent degradation in visual inspections). All renders have a maximum probability of belonging to degradation level B at year 3, but each exposure condition shows a distinct probability: $\mathrm{P}=50.4 \%, 40.3 \%$ and $39 \%$ for unfavourable, standard and favourable conditions of exposure to driven-rain, respectively. Renderings with current level of protection reach the maximum probability (44.1\%) of belonging to degradation level $\mathrm{C}$ at around year 8 . The corresponding values for renders with poor and good levels of protection are $51.1 \%$ and $55.8 \%$, respectively, both at around year 9 . Renders with good levels of protection reach the maximum probability of belonging to degradation level $\mathrm{D}$ at around year $17(\mathrm{P}=27.9 \%)$. Renders with current level of protection reach this peak $(\mathrm{P}=44.7 \%)$ at year 18 . The corresponding value for renders with poor level of protection occurs at around year $20(\mathrm{P}=41.6 \%)$. These results apparently contradict expectations and are further analysed in the discussion section. 


\subsubsection{Pollution}

Atmospheric pollutants contribute to the physical and visual degradation of materials and building components, especially of materials of porous nature such as render (Kus and Nygren, 2002). The rain associated with these pollutants is acid and aggressive to building materials, which promotes their deterioration, especially if they are cement-based (Zivica and Bajza, 2001). In this study three categories concerning exposure to pollutant sources were considered, according to the study of Gaspar (2009): i) favourable - for environments with no exposure to industrial or vehicle pollution sources (in countryside or seaside areas), outside of the main urban centres; ii) normal for urban areas with moderate traffic (residential areas outside the main roadways); iii) unfavourable - concerning the areas located near main roadways. Figures 9 and 10 present the mean time of permanence in each degradation state as a function of exposure to pollutant sources and the probabilistic distribution of the degradation condition as a function of age and exposure to pollutant sources, respectively. In this study, only six case studies have favourable exposure to pollutant sources and none of them presents degradation level E. For that reason, the model computed using Markov chains assumes that it is necessary a long period of time for a render with favourable exposure to pollutant sources to change from level D to level E. Renders with favourable exposure to pollutant sources reach the maximum probability $(\mathrm{P}=69 \%)$ of belonging to degradation level B after year 2. Renders with unfavourable and normal exposure to pollutant sources have a maximum probability of belonging to degradation level B of $39.7 \%$ and $43 \%$ respectively, both at around year 3. Renders with normal exposure to pollutant sources reach the maximum probability $(\mathrm{P}=54.1 \%)$ of belonging to degradation level $\mathrm{C}$ at around year 9 . The corresponding values for renders with unfavourable and favourable exposure to pollutant sources are $\mathrm{P}=42.5 \%$ and $56 \%$, respectively, both at around year 8 .

\section{Discussion of the results}

In this study, Markov chains were used to model the rate of transition between five condition levels of render. The results are also broken down according to the most influential environmental factors and estimate the time after which they will be unable to meet the performance requirements for 
which they were designed.

The sample studied was successfully modelled by Markov chains processes, despite the relatively short sample studied, thus demonstrating the applicability of these methods for durability assessment and service life estimation of building elements. However, the results obtained should be considered cautiously as they may be subjected to distortions due to the sample size. As with all statistical methods, the more data is available, the more accurate the results obtained are, with fewer samplespecific outcomes.

The overall results confirm previous data on the degradation of render, but deal with the uncertainty associated to the action of environmental agents in the durability of the material. In fact, all results thus obtained represent probabilistic distributions rather than deterministic values. Through this method it is possible to conclude that external renders go through the early stages of degradation in only 5 years (conditions A and B) as shown in Figures 1 and 2. The relatively short time of permanence in levels $\mathrm{A}$ and $\mathrm{B}$ and the longer periods in levels $\mathrm{C}$ and $\mathrm{D}$ confirm the results from previous research according to which renders experience a fast initial process of degradation, when early defects become evident and are eventually corrected if they are deemed to be serious, followed by a maturing phase when environmental actions slowly and steadily alter the performance of render, but whose effects become visually apparent only some years after (Gaspar and de Brito, $2005,2008 \mathrm{a}$ ). These results can also be explained by the greater sensitivity of the model to detect early changes in the surface of the render as opposed to the degree of degradation that is required for renders to change between levels $\mathrm{C}, \mathrm{D}$ and $\mathrm{E}$.

Figure 11 shows the mean time of permanence in each degradation state according to the environmental factors analysed. From all factors studied, distance from the sea is the one in which a distinction is more noticeable at early degradation levels. For levels $\mathrm{B}$ and $\mathrm{C}$ there are no striking overall differences in the results. Finally, for level D - considered to be the limit for service life of render - it is possible to identify the favourable effects of non-polluted environments as renders remain in condition $\mathrm{D}$ and do not progress to condition $\mathrm{E}$. Renders on buildings situated off coast 
also remain less time in level $\mathrm{D}$ of degradation, which clearly suggests that marine influence is the most influential environmental stress factor for their service life.

\subsection{Breakdown of results for the environmental factors}

Concerning the life cycle of external renders, several authors have developed different methods to estimate the service life of this type of coating. Gaspar (2009) predicted a service life of 15 - 20 years for render façades for a maximum degradation level between $20 \%$ and $30 \%$. Shohet and Paciuk (2004) took two distinct levels of requisites: for the most stringent one the service life of render façades was estimated as 15 years (with a range of 12 - 19 years); for a lower level of requirement the service life rose to 23 years (with a range of 19-27 years). Regarding these results, in this study, a life horizon of 40 years (twice the average value of estimated service life of external render) was considered. The results shown in Table 4 confirm the empirical perception that distance from the sea directly affects the durability of external render. In fact, renders located less than $3.5 \mathrm{~km}$ from the sea show a higher probability of being in the highest degradation levels $(\mathrm{P}=56.5 \%$ of being in either condition levels $\mathrm{D}$ or $\mathrm{E}$ ). If one considers the mean permanence time of renders in each condition level, as well as the moment when transitions occur between condition states, it is possible to obtain a more direct indication of the effects of environmental agents in their service life. In particular, it is important to analyse the influence of environmental agents in the occurrence of condition $\mathrm{D}$, regarded as the service life threshold beyond which repair work is required. Proximity from the sea also greatly increases the probability of condition D, with a peak before year 15, when compared to cases distant from the sea as shown in Figure 4.

Table 5 presents the probability of belonging to each degradation condition as a function of exposure to damp. Render with low exposure has a higher probability of being in the lowest degradation levels ( $\mathrm{A}$ and $\mathrm{B}$ ) $\mathrm{P}=39.4 \%$. Render with severe exposure has a higher probability of being in degradation levels $\mathrm{C}$ and $\mathrm{D}, \mathrm{P}=91 \%$, and has null probability of being in the lowest levels. In fact, defects like dark patches of damp and microorganisms and algae growth are associated with a higher exposure to damp, e.g. higher degradation levels. Damp in render also promotes cracking and stresses in the internal structure of the render induced by dimensional 
variations. Severe damp exposure tends to anticipate the occurrence of condition D in three years and in more than five years, as compared to renders with average and low damp exposure respectively. These results are coherent and suggest that renders with high exposure to damp are more prone to higher degradation levels and require constant actions of maintenance and repair. Noteworthy is the fact that exposure to damp does not significantly increase the probability of occurrence of the most severe degradation levels, which seems to confirm previous indications that dampness, especially when connected to staining, does not directly determine the end of durability for render (Gaspar and de Brito, 2008a,b).

The differences between renders under different exposure to wind-driven rain are significantly lower than observed for the two previous exposures. Table 6 illustrates the probability of belonging to each of the degradation conditions as a function of the level of protection of a facade. Facades with a poor level of protection have the highest probability of being in degradation level $\mathrm{D}$ and a relatively high probability of being in levels $\mathrm{C}$ and $\mathrm{E}$. Facades with standard level of protection have similar probabilities of being in all degradation levels, even though this probability is lower for level D. Facades with good level of protection have a high probability of being in degradation level $\mathrm{C}$. These results challenge the perception that higher protection levels lead to lower degradation levels. Additional research was thus required in order to better understand this phenomenon. Figure 12 shows the analysis of the various degradation levels according to both exposure to damp and protection level of the facades. Facades with severe exposure to damp are more prone to have higher levels of degradation when the protection level of the facade is poor. In the sample analysed, facades with good protection level and severe exposure to damp only belong to degradation level C. Facades with low exposure to damp have a higher probability of belonging to lowest levels of degradation (levels A and B). Figure 13 shows the analysis of the various degradation levels according to both distance from the sea and protection level of the facades. Facades located less than $3.5 \mathrm{~km}$ from the sea and with poor protection level of the facade have a higher probability (50\%) of belonging to degradation level E. Likewise, facades on the coast have a lower probability of belonging to degradation level A, i.e. only facades 
with current level of protection can be in this degradation level (with a probability of $13 \%$ ).

Facades located more than $3.5 \mathrm{~km}$ from the sea are more prone to be in degradation levels A and B, with a global probability of $37 \%$ for facades with poor protection level, $50 \%$ for facades with current protection level and 30\% for facades with good protection level. It is thus concluded that, although the facade protection level may not have correctly modelled the effects of wind driven rain (it was in fact the only environmental factor that was indirectly assessed), it is proven that lower levels of protection of the façade do enhance the deleterious effects of other environmental factors, while better protection levels delay these degradation effects.

It was assumed that exposure to pollutants is directly related to the proximity to urban roads. However, distance from pollutant sources, and particularly the most polluted roads of a city, does not necessarily mean a lower exposure to pollutants. In fact, depending on the diffusion and transport of airborne pollutants, even seemingly sheltered streets may suffer the effects of pollution. There are many uncertainties regarding this factor, namely due to the absence of detailed maps concerning the environmental quality of urban air. Table 7 shows the probability of belonging to each degradation condition as a function of exposure to pollutants. The results suggest a clear distinction between the facades in urban areas and in the countryside. Renders with favourable exposure to pollutants have (a) a higher probability of being in degradation levels B and $\mathrm{C}, \mathrm{P}=66.6 \%$, (b) low probability of being in level $\mathrm{D}(\mathrm{P}=16.7 \%)$ and (c) null probability of being in level E. These results suggest that end-of-life conditions are not reached, within the time horizon considered, when pollution is not present. Renders with current and unfavourable exposure to pollutants are more prone to higher degradation levels, and are the most likely to present degradation level D and $\mathrm{E}, \mathrm{P}=45.8 \%$. Service life variations related to exposure to pollutants are not as clear as the results for other environmental agents. However, it is possible to identify a pattern along which buildings with low exposure to pollutants do not reach condition level E. For unfavourable conditions of exposure to pollutants, variations in degradation do not occur at early stages of service life, as shown in Figures 9 and 10. In fact, all renderings seem to pass trough transitions of condition $\mathrm{A}$ to $\mathrm{B}$ and $\mathrm{B}$ to $\mathrm{C}$ in the first five years of service life. 
However, transition between levels $\mathrm{C}$ and $\mathrm{D}$ is faster for unfavourable conditions. At year 5, in renderings with unfavourable exposure conditions, degradation level D tends to occur 1 to 2 years earlier and increase at a more rapid rate than in other renderings, which suggests that unfavourable conditions tend to accelerate the degradation process.

\subsection{Modelling the end of service}

Table 8 shows the maximum probability of belonging to degradation level D (which marks the end of service life). The overall sample reaches the maximum probability of belonging to level D $(38.5 \%)$ at year 19. The probability of the renderings being in Level D or E (i.e. unacceptable) reaches $50 \%$ at year 14 . Concerning distance from the sea, it is found that for coast locations this probability is reached after 10 years, while for interior locations it takes 23 years. Facades with severe exposure to damp reach the probability of $50 \%$ of belonging to level D or E at year 10 , sooner than facades with low and moderate exposure (this probability is reached after 17 and 11 years, respectively). Facades with good protection level reach the probability of $50 \%$ of being in degradation level D or E after 15 years. For facades with poor protection level, this probability is reached after 14 years and for facades with current exposure, this probability is reached after 12 years. This result seemingly contradicts the empirical knowledge, regardless of the fact that it has been shown that the level of protection of the façade has direct influence on the severity of other environmental agents. Concerning exposure to pollutants, in the sample analysed there are no cases with favourable exposure belonging to degradation level E. For unfavourable exposure, the probability of $50 \%$ of belonging to level $\mathrm{D}$ or $\mathrm{E}$ is reached at year 11 , while for normal exposure it takes 15 years and for favourable exposure 17 years.

\subsection{Application of the results to maintenance planning}

The probabilistic distribution of condition over time, as shown on Table 3 , can also be interpreted as an assessment of risk of not performing as expected due to degradation. Thus, by establishing the threshold of acceptable risk, a stakeholder may visualize the need for repair based on the probabilistic analysis of a set of data. To illustrate this concept, it can be 
considered that "high", "average" and "low" probability of a given condition correspond to "high", "average" and "low" risk associated to the consequences of defects and the cost of repair and thus produce an indication of the urgency to maintenance and repair actions. Low risk (no action required apart from monitoring) is considered to correspond to $\mathrm{P}>75 \%$ of belonging to either condition levels " $\mathrm{A}$ " or " $\mathrm{B}$ ". High risk (a need to undertake extensive repair) is considered to correspond to $\mathrm{P}>25 \%$ of belonging to condition levels " $\mathrm{E}$ " and average risk corresponds to the intermediate states. From such interpretation, recommendations could be made in order to i) monitor the render facade until year five; ii) perform light maintenance actions until year 10; iii) perform repair works until year 15; and iv) consider replacement of the render from year 15 onwards, subject to on-site confirmation of its condition.

\section{Discussion and conclusions}

This study focuses on a probabilistic analysis of the degradation condition of render facades and its relationship with the most influential environmental factors. In service life prediction it is important to know the rate of transition between degradation states. This assists with predicting the behaviour of the render facades and estimating when will be unable to meet their performance requirements. Future performance was predicted using Markov chains, which are a stochastic model widely employed in studies concerning the modelling of durability that requires little information in the calibration procedure. However, the application of Markov chains in degradation models has some limitations. The results from this study allow the following main qualitative conclusions: (a) render facades nearer the sea have higher degradation levels, and consequently, a shorter service life by six years i.e. one third of the expected service life; (b) render with high exposure to damp are more prone to higher degradation levels and may experience service life reductions of three to five years; (c) render with current protection level (current exposure to the combined action of rain and wind) offer the best performance; (d) the distinction between cases located in urban areas and in the countryside was clear; but exposure to pollutants seems not to directly affect the durability of external renders (for the sample analysed).

These results confirm that render facades are particularly sensitive to the effects of environmental 
agents (Gaspar and Brito, 2008). The values obtained using a Markov chains model are coherent and supported by the literature. The general model applied to global sample reveals that rendered facades have a maximum probability of reaching the end of their service life between 15 and 16 years. Similar results are obtained by Shohet and Paciuk (2004) who obtained an estimated service life for rendered facades of 15 years (with a range between 12 and 19 years).

Concerning the environmental conditions, it is found that renders in coastal locations reach the end of their service life after 10 years and renders with unfavourable exposure to pollutants present a probability of $50 \%$ of belonging to level $\mathrm{D}$ or $\mathrm{E}$ at year 11 . These values are within the range of results obtained by Shohet and Paciuk (2006), which reveals that the failure of render due to air pollution occurs after between 11 and 19 years and failure of render due to proximity of a marine environment occurs after between 7 and 13 years.

The presented method,based on Markov chains, is therefore capable of providing indications about a complex phenomenon such as facade degradation. It can provide information on the synergy between the degradation agents and the degradation levels. In particular, it shows a probabilistic distribution of the condition of the render façades over time and as a function of the surrounding environmental characteristics, the average time of permanence in each condition until the transition to the next degradation level and indications of the effect of degradation on the durability of renders that may be applied to maintenance procedures. Such information can help to define - in a rational and technically informed way - a set of maintenance strategies throughout the building life cycle. Furthermore, whenever information is available on the durability of the various components of the building, it is also possible to establish joint maintenance strategies for different parts and elements of the building. Finally, stochastic models such as Markov chains provide crucial information in the context of insurance policies. This allows an evaluation of the risk of failure and provides new information about the most probable failure time of building elements based on their characteristics.

\section{Acknowledgements}

Comment [E3]: The discussion of Chew and Ping is not relevant or appropriate here. Therefore, it has been deleted.

Comment [AS4]: The authors agree with the editor's option and have removed the discussion. 
The authors gratefully acknowledge the support of the ICIST Research Institute, IST, Technical University of Lisbon and of the FCT (Foundation for Science and Technology).

\section{References}

Aikivuori, A.M. (1999) ‘Critical loss of performance - what fails before durability’, 8DBMC, Durability of Building Materials and Components, Vancouver, Canada, pp. 1369-1376.

Atkinson, B.; Snape, P. (1999) 'Mapping rainfall distribution characteristics on facades using surface deposit geometry', 8DBMC, Durability of Building Materials and Components, Vancouver, Canada, pp. 943-55.

Augenbroe, G.L.M., Park, C.-S. (2002) ‘Towards a Maintenance Performance Toolkit for GSA', Interim Report submitted to GSA, Georgia Institute of Technology, Atlanta, USA. Basso, N., Garavaglia, E., Sgambi, L. (2012) 'Selective maintenance planning based on a Markovian approach', IALCCE - International Association for life-cycle Civil Engineering, 3rd International Symposium on Life-Cycle Civil Engineering 2012, October 3-6, Vienna, Austria, pp. 435-441. Bladty, M.; Sorensen, M. (2009) 'Efficient estimation of transition rates between credit ratings from observations at discrete time points', Quantitative Finance, 9(2), pp. 147-160.

Bocchini, P.; Saydam, D.; Frangopol, D.M. (2013) 'Efficient, accurate, and simple Markov chain model for the life-cycle analysis of bridge groups' Structural Safety, 40, pp. 51-64. Bonshor, R.; Bonshor, L. (2001) 'Cracking in buildings’, BRE, London, 106 p. BSI 7543, 1992. Guide to durability of buildings and building elements. British Standards Institution, London, United Kingdom.

Carrington, P.J.; Scott, J.; Wasserman, S. (eds.) (2005) 'Models and methods in social network analysis', $1^{\text {st }}$ Edition, Cambridge University Press, New York, USA.

Cheung, M.S.; Kyle, B.R. (1996) 'Service life prediction of concrete structures by reliability analysis', Construction and Building Materials, 10 (1), pp. 45-55.

Chew, M.Y.L.; Ping, T.P. (2003) ‘Staining of facades'. World Scientific Publishing, Singapore. 
Chew, M.Y.L.; Tan, P.P. (2003) 'Facade staining arising from design features', Construction and Building Materials, 17 (3), pp. 181-187.

Choi, E.C. (1999), 'Wind driven rain on building facades and the driving rain index', Wind Engineering and Industrial Aeronautics, 79 (1-2), pp. 105-22.

Costa, E.A.L.; Campos, V.P.; da Silva Filho; L.C.P.; Greven, H.A. (2009) 'Evaluation of the aggressive potential of marine chloride and sulfate salts on mortars applied as renders in the Metropolitan Region of Salvador - Bahia, Brazil', Journal of Environmental Management, 90 (2), pp. 1060-1068.

Cox, D.R.; Miller, H.D. (1965) 'The Theory of Stochastic Processes', $1^{\text {st }}$ Edition, Chapman and Hall, London, UK.

Durango-Cohen, P.L.; Madanatz, S.M. (2006) 'Optimization of inspection and maintenance decisions for infrastructure facilities under performance model uncertainty: a quasi-Bayesian approach', Transportation Research Part A: Policy and Practice, 42 (8), pp. 1074-1085. Ellingwood, B.R. (2005) 'Risk-informed condition assessment of civil infrastructure: state of practice and research issues', Structure and Infrastructure Engineering: Maintenance, Management, Life-Cycle Design and Performance, 1 (1), pp. 7-18.

Flores-Colen, I.; de Brito, J.; de Freitas; V.P. (2009) 'Expected render performance assessment based on impact resistance in situ determination', Construction and Building Materials, 23 (9), pp. 2997-3004.

Flores-Colen, I.; de Brito, J. (2009) ‘A systematic approach for maintenance budgeting of buildings facades based on predictive and preventive strategies'. Construction and Building Materials, 24(9), pp. 1718-1729.

Frangopol, D.M.; Kallen, M.-J.; Noortwijk, J.M.V. (2004) 'Probabilistic models for life-cycle performance of deteriorating structures: review and future directions', Progress in Structural Engineering and Materials, 6 (4), pp. 197-212.

Freitas, V.P.; Sousa, M.; Abrantes, V. (1999) 'Survey of the durability of facades of 4.000 dwellings in northern Portugal', 8DBMC, Durability of Building Materials and Components, Vancouver, Canada, pp. 1040-1050. 
Garavaglia, E.; Gianni, A.; Molina, C. (2004) 'Reliability of porous materials: two stochastic approaches', Journal of Materials in Civil Engineering, 16 (5), pp. 419-426.

Gaspar, P.; de Brito, J. (2005) 'Mapping defect sensitivity in external mortar linings', Construction and Building Materials, 19 (8), pp. 571-578.

Gaspar, P.L. and de Brito, J. (2008a) ‘Service life estimation of cement-rendered façades', Building Research and Information, 36 (1), pp. 44-55.

Gaspar, P.L.; de Brito, J. (2008b) 'Quantifying environment effects on cement-rendered facades: a comparison between different degradation indicators', Building and Environment, 43 (11), pp. 1818-1828.

Gaspar, P. (2009) 'Service life of constructions: development of a methodology to estimate the durability of construction elements. Application to renders in current buildings', $\mathrm{PhD}$ thesis, Instituto Superior Técnico, Lisbon, Portugal, [In Portuguese].

Gaspar, P.L.; de Brito, J. (2011) 'Limit states and service life of cement renders on façades', Materials in Civil Engineering, 23 (10), pp. 1393-1404.

Handel R.V. (2008) ‘Hidden Markov models', Lecture Notes, Princeton University, New Jersey, USA.

Hawk, H.; Small, E.P. (1998) 'The BRIDGIT bridge management system', Structural Engineering International, IABSE, 8(4), pp. 309-314.

Hansen, E.J.P.; Ekman, T.; Hansen, K.K. (1999) 'Durability of Cracked Fibre Reinforced Concrete Structures Exposed to Chlorides', 8DBMC, Durability of Building Materials and Components, Vancouver, Canada, pp. 280-289.

Higham, D.; Higham, N. (2005) 'MATLAB guide'. $2^{\text {nd }}$ Edition, Society for Industrial Mathematics, Philadelphia, USA.

Hossain, K.M.A.; Lachemi, M.; Şahmaran, M. (2009) 'Performance of cementitious building renders incorporating natural and industrial pozzolans under aggressive airborne marine salts', Cement and Concrete Composites, 31 (6), pp. 358-368.

ISO 15686-1:2000. Building and constructed assets: Service life planning - Part 1: General principles. Geneva: International Standard Organization; 2000. 
Kalbfleisch, J.D.; Lawless, J.F. (1985) 'The analysis of panel data under a Markov assumption', Journal of the American Statistical Association, 80 (392), pp. 863-871.

Kallen, M.J. (2009) 'A comparison of statistical models for visual inspection data', Safety, Reliability and Risk Structures, Infrastructures and Engineering Systems. Tenth International Conference on structural safety and reliability (ICOSSAR), Osaka, Japan, pp. 3235-3242. Kus, H.; Nygren, K. (2002) 'Microenvironmental characterization of rendered autoclaved aerated concrete', Building Research and Information, 30 (1), pp. 25-34.

Lawless J.F. (1982) 'Statistical models and methods for lifetime data', $1^{\text {st }}$ Edition, John Wiley and Sons, New York, USA.

Lounis, Z.; Vanier, D.J. (2000) 'A multi-objective and stochastic system for building maintenance management', Computer-Aided Civil and Infrastructure Engineering, 15 (5), pp. 320-329.

Lourenço, P.B.; Luso, E.; Almeida, M.G. (2006) 'Defects and moisture problems in buildings from historical city centres: a case study in Portugal', Building and Environment, 41 (2), pp. 223-234. Mc Duling, J.J. (2006) 'Towards the development of transition probability matrices in the Markovian model for the predicted service life of buildings', $\mathrm{PhD}$ Thesis in Civil Engineering, Faculty of Engineering, Built Environment and Information Technology, University of Pretoria, Pretoria.

Morcous, G.; Lounis, Z.; Mirza, M.S. (2003) 'Identification of environmental categories for Markovian deterioration models of bridge decks', Journal of Bridge Engineering, 8 (6), pp. 353-361. Morcous, G.; Lounis, Z. (2005) 'Maintenance optimization of infrastructure networks using genetic algorithms', Automation in Construction, 14 (1), pp. 129-142.

Moser, K. (2003) 'Engineering design methods for service life planning - state of the art', International Workshop on Management of Durability in the Building Process, Politecnico di Milano, Milan, Italy, paper 40.

Moser, K. (2004) 'Engineering design methods for service life prediction. Performance Based Methods for Service Life Prediction - State of the Art Report, Part B', CIB Report 294, 107 p. National Statistics Institute (INE). National statistics - census 2001

$<$ http://www.ine.pt/prodserv/quadro/mostraquadro.asp $>$ [in Portuguese] 

maintenance of existing steel structures', $6^{\text {th }}$ International Symposium Steel Bridges, European Convention for Construction Steelwork, Prague, Czech Republic, pp. 206-215.

Norris, J.R. (1997), ‘Markov Chains', $1^{\text {st }}$ Edition, Cambridge University Press, Cambridge, UK. Ortiz-García, J.J.; Seósamh, B.C.; Martin, S.S. (2006) ‘Derivation of transition probability matrices for pavement deterioration modeling', Journal of Transportation Engineering, 132 (2), pp. 141-161. Parnham, P. (1997) 'Prevention of premature staining of new buildings', $1^{\text {st }}$ Edition, E \& FN Spon, Spon Press, London, UK.

Parzen, E. (1962) ‘Stochastic Processes’, Holden Day, San Francisco, USA.

Paulo, P.V.; Branco, F.; de Brito, J. (2013) ‘BuildingsLife: a building management system’, Structure and Infrastructure Engineering: Maintenance, Management, Life-Cycle Design and Performance, DOI: 10.1080/15732479.2012.756919.

Rirsch, E.; MacMullen, J.; Zhang, Z. (2011) 'Evaluation of mortar samples obtained from UK houses treated for rising damp', Construction and Building Materials, 25 (6), pp. 2845-2850. Robelin, C.A.; Madanat, S. (2007) 'History-dependent bridge deck maintenance and replacement optimization with Markov decision process', Journal of Infrastructure Systems, 13 (3), pp. 195-201. Rodrigues, M.F.S., Cardoso Teixeira, J.M.; Cardoso, J.C.P.; Batel Anjos, A.J. (2013) ‘Envelope index evaluation model of existing buildings'. Civil Engineering and Environmental Systems, 30 (1), pp. 26-39.

Sabbioni, C.; Zappia, G.; Riontino, C.; Blanco-Varela, M.T.; Aguilera, J.; Puertas, F.; van Balen, K.; Toumbakari, E.E. (2001) 'Atmospheric deterioration of ancient and modern hydraulic mortars', Atmospheric Environment, 35 (3), pp. 539-548. Shohet, I.; Paciuk, M. (2004) 'Service life prediction of exterior cladding components under standard conditions. Journal of Construction Management and Economics, 22 (10): 1081-1090. Shohet, I.M.; Paciuk, M. (2006) 'Service life prediction of exterior cladding components under failure conditions'. Journal of Construction Management and Economics, 24 (2), pp. 131-148. 
Silva, A.; de Brito, J.; Gaspar, P. (2011) 'Service life prediction model applied to natural stone wall claddings (directly adhered to the substrate)', Construction and Building Materials, 25 (9), pp. 3674-3684.

Silva, A.; de Brito, J.; Gaspar, P. (2012) 'Life cycle of building's facades: service life prediction of natural stone wall claddings using the factor method', IALCCE - International Association for life-cycle Civil Engineering, Third international Symposium on Life-Cycle Civil Engineering, October 3-6, Vienna, Austria, pp. 1709-1715.

Silva, A.; Dias, J.L.R.; Gaspar, P.L.; de Brito, J. (2013) 'Statistical models applied to service life prediction of rendered façades', Automation in Construction, 30, pp. 151-160.

Singer, B. (1981) 'Estimation of nonstationary Markov chains from panel data', Sociological Methodology, 12, pp. 319-337.

Soroka, I.; Carmel, D. (1987) 'Durability of external renderings in a marine environment durability of building materials', Elsevier Science Publishers, Amsterdam, Netherlands, pp. 6172.

Straub, A. (2003) 'Using a condition-dependent approach to maintenance to control costs and performances', Facilities Management, 1 (4), pp. 380-395.

Thompson, P.D.; Small, E.P.; Johnson, M.; Marshall, A.R. (1998) 'The Pontis bridge management system', Structural Engineering International, IABSE, 8 (4), pp. 303-308.

Van Winden, C.; Dekker, R. (1998) 'Rationalization of building maintenance by Markov decision models: a pilot case study', Journal of Operation Research Society, 49 (9), pp. 928 935.

Zivica, V.; Bajza, A. (2001) 'Acidic attack of cement based materials - a review: Part 1. Principle of acidic attack', Construction and Building Materials, 15 (8), pp. 331-340.

Zivica, V.; Palou, M.T.; Krizma, M.; Bagel, L. (2012) 'Acidic attack of cement based materials under the common action of high, ambient temperature and pressure', Construction and Building Materials, 36, pp. 623-629. 
Table 1- Proposed classification of degradation condition of renderings

Level A

$$
\text { Condition level }
$$

Physical and visual assessment

Severity of degradation

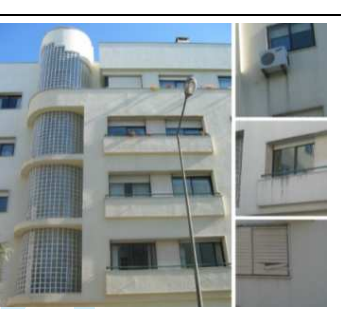

Complete mortar surface with no deterioration. Surface even and uniform. No visible cracking or cracking $\leq 0.1 \mathrm{~mm}$. Uniform colour and no dirt. No detachment of elements.

Level B

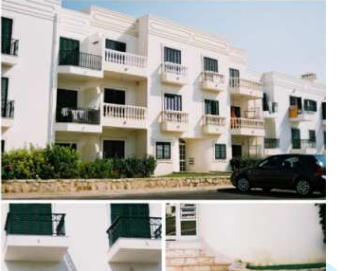

Non-uniform mortar surface with likelihood of hollow localized areas determined by percussion, but no signs of detachment. Small cracking $(0.25 \mathrm{~mm}$ to $1.0 \mathrm{~mm})$ in localized areas. Changed in the general colour of the surface. Eventual presence of microorganisms.

Localized detachments or

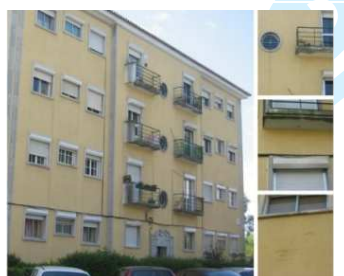

perforations of the mortar. Hollow sound when tapped. Detachments only in the socle. Easily visible cracking ( $1.0 \mathrm{~mm}$ to $2.0 \mathrm{~mm}$ ). Dark patches of damp and dirt, often with microorganisms and algae.

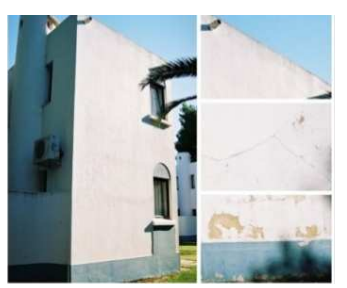

Incomplete mortar surface due to detachments and falling of mortar patches. Wide or extensive cracking ( $\geq 2 \mathrm{~mm}$ ). Very dark patches with

15 to

Level D probable presence of algae.

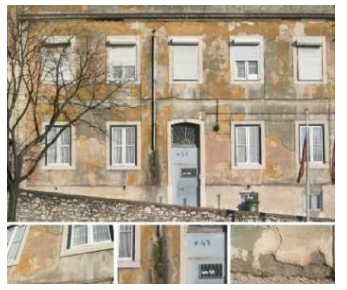

Incomplete mortar surface due to detachments and falling of mortar patches. Wide or extensive cracking ( $\geq 2 \mathrm{~mm}$ ). Very dark patches with

Level E probable presence of algae. 
Table 2 - Analysis of the efficiency of the model obtained with Markov chains

\begin{tabular}{c|c|c|c}
\hline Degradation condition & Observed & Predicted & Mean percentage error (\%) \\
\hline Level A & 14 & 12.6 & 10.2 \\
\hline Level B & 18 & 17.8 & 1.3 \\
\hline Level C & 31 & 28.6 & 7.6 \\
\hline Level D & 15 & 17.3 & 15.3 \\
\hline Level E & 22 & 22.7 & 3.4 \\
\hline
\end{tabular}


Table 3 - Probability of belonging to a condition level as a function of age

\begin{tabular}{c|c|c|c|c|c}
\hline \multirow{2}{*}{$\begin{array}{c}\text { Range in } \\
\text { years }\end{array}$} & \multicolumn{5}{|c}{ Probability of belonging to a condition level } \\
\cline { 2 - 6 } & Level A & Level B & Level C & Level D & Level E \\
\hline$[0: 5]$ & $45.87 \%$ & $31.82 \%$ & $19.14 \%$ & $2.91 \%$ & $0.28 \%$ \\
\hline$] 5: 10]$ & $4.70 \%$ & $22.26 \%$ & $49.57 \%$ & $19.07 \%$ & $4.40 \%$ \\
\hline$] 10: 15]$ & $0.63 \%$ & $7.17 \%$ & $44.57 \%$ & $33.06 \%$ & $14.56 \%$ \\
\hline$] 15: 20]$ & $0.08 \%$ & $1.99 \%$ & $31.41 \%$ & $38.14 \%$ & $28.38 \%$ \\
\hline$[20: 25]$ & $0.01 \%$ & $0.52 \%$ & $20.26 \%$ & $36.48 \%$ & $42.73 \%$ \\
\hline$] 25: 30]$ & $0.00 \%$ & $0.13 \%$ & $12.62 \%$ & $31.52 \%$ & $55.72 \%$ \\
\hline$] 30: 35]$ & $0.00 \%$ & $0.03 \%$ & $7.75 \%$ & $25.61 \%$ & $66.60 \%$ \\
\hline$] 35: 40]$ & $0.00 \%$ & $0.01 \%$ & $4.74 \%$ & $20.00 \%$ & $75.25 \%$ \\
\hline
\end{tabular}


Table 4 - Probability of belonging to a condition level as a function of distance from the sea

\begin{tabular}{c|c|c|c|c|c}
\hline \multirow{2}{*}{ Distance from the sea } & \multicolumn{5}{|c}{ Probability of belonging to a condition level } \\
\cline { 2 - 6 } & Level A & Level B & Level C & Level D & Level E \\
\hline$>3.5 \mathrm{~km}$ & $16.9 \%$ & $20.8 \%$ & $31.2 \%$ & $11.7 \%$ & $19.5 \%$ \\
\hline$\leq 3.5 \mathrm{~km}$ & $4.3 \%$ & $8.7 \%$ & $30.4 \%$ & $26.1 \%$ & $30.4 \%$ \\
\hline
\end{tabular}


Table 5 - Probability of belonging to a condition level as a function of exposure to damp

\begin{tabular}{c|c|c|c|c|c}
\hline \multirow{2}{*}{ Exposure to damp } & \multicolumn{5}{|c}{ Probability of belonging to a condition level } \\
\cline { 2 - 6 } & Level A & Level B & Level C & Level D & Level E \\
\hline Low & $18.2 \%$ & $21.2 \%$ & $34.8 \%$ & $7.6 \%$ & $18.2 \%$ \\
\hline Moderate & $8.7 \%$ & $17.4 \%$ & $13.0 \%$ & $21.7 \%$ & $39.1 \%$ \\
\hline Severe & $0.0 \%$ & $0.0 \%$ & $45.5 \%$ & $45.5 \%$ & $9.1 \%$ \\
\hline
\end{tabular}


Table 6 - Probability of belonging to a condition level as a function of facades protection

\begin{tabular}{c|c|c|c|c|c}
\hline \multirow{2}{*}{ Facades protection } & \multicolumn{5}{|c}{ Probability of belonging to a condition level } \\
\cline { 2 - 6 } & Level A & Level B & Level C & Level D & Level E \\
\hline Poor & $13.0 \%$ & $17.4 \%$ & $30.4 \%$ & $17.4 \%$ & $21.7 \%$ \\
\hline Current & $20.8 \%$ & $20.8 \%$ & $20.8 \%$ & $16.7 \%$ & $20.8 \%$ \\
\hline Good & $10.0 \%$ & $16.7 \%$ & $40.0 \%$ & $10.0 \%$ & $23.3 \%$ \\
\hline
\end{tabular}


Table 7 - Probability of belonging to a condition level as a function of exposure to pollutants

\begin{tabular}{c|c|c|c|c|c}
\hline \multirow{2}{*}{ Exposure to pollutants } & \multicolumn{5}{|c}{ Probability of belonging to a condition level } \\
\cline { 2 - 6 } & Level A & Level B & Level C & Level D & Level E \\
\hline Favourable & $16.7 \%$ & $33.3 \%$ & $33.3 \%$ & $16.7 \%$ & $0.0 \%$ \\
\hline Normal & $12.9 \%$ & $17.1 \%$ & $34.3 \%$ & $11.4 \%$ & $24.3 \%$ \\
\hline Unfavourable & $16.7 \%$ & $16.7 \%$ & $20.8 \%$ & $25.0 \%$ & $20.8 \%$ \\
\hline
\end{tabular}


Table 8 - Analysis of the maximum probability of belonging to degradation level D (end of service life)

\begin{tabular}{|c|c|c|c|c|c|}
\hline & & $\begin{array}{l}\text { Maximum } \\
\text { probability of } \\
\text { belonging to } \\
\text { degradation } \\
\text { level D }\end{array}$ & $\begin{array}{c}\text { Age at reaches } \\
\text { the maximum } \\
\text { probability of } \\
\text { belonging to } \\
\text { degradation level } \\
\text { D (years) }\end{array}$ & $\begin{array}{c}\text { Ratio between the } \\
\text { maximum } \\
\text { probability of } \\
\text { belonging to } \\
\text { degradation level D } \\
\text { for the } \\
\text { characteristics } \\
\text { analysed and for } \\
\text { overall sample }\end{array}$ & $\begin{array}{l}\text { Ratio between the age at } \\
\text { reaches the maximum } \\
\text { probability of belonging } \\
\text { to degradation level D } \\
\text { for the characteristics } \\
\text { analysed and for overall } \\
\text { sample }\end{array}$ \\
\hline & $\begin{array}{l}\begin{array}{l}\text { Overall } \\
\text { sample }\end{array} \\
\end{array}$ & $38.47 \%$ & 19 & - & - \\
\hline \multirow{2}{*}{$\begin{array}{l}\text { Distance } \\
\text { from the sea }\end{array}$} & $>3.5 \mathrm{~km}$ & $5.38 \%$ & 16 & 0.14 & 0.84 \\
\hline & $\leq 3.5 \mathrm{~km}$ & $46.85 \%$ & 16 & 1.22 & 0.84 \\
\hline \multirow{3}{*}{$\begin{array}{l}\text { Exposure to } \\
\text { damp }\end{array}$} & low & $27.34 \%$ & 19 & 0.71 & 1 \\
\hline & moderate & $47.12 \%$ & 17 & 1.22 & 0.89 \\
\hline & severe & $62.83 \%$ & 22 & 1.63 & 1.16 \\
\hline \multirow{3}{*}{$\begin{array}{l}\text { Protection } \\
\text { level of the } \\
\text { facades }\end{array}$} & poor & $41.64 \%$ & 20 & 1.08 & 1.05 \\
\hline & current & $44.72 \%$ & 18 & 1.16 & 0.95 \\
\hline & good & $27.90 \%$ & 17 & 0.73 & 0.89 \\
\hline \multirow{3}{*}{$\begin{array}{l}\text { Exposure to } \\
\text { pollutants }\end{array}$} & unfavourable & $52.04 \%$ & 18 & 1.35 & 0.95 \\
\hline & normal & $32.36 \%$ & 18 & 0.84 & 0.95 \\
\hline & favourable* & - & - & - & - \\
\hline \multicolumn{6}{|c|}{$\begin{array}{l}\text { * In the sample analysed, there is no case studies belonging to degradation level E with favourable exposure to } \\
\text { pollutants. For this reason, it is impossible to model the transition between levels D and E. The values are not } \\
\text { shown because for favourable exposure to pollutants it is not possible to define a maximum probability of } \\
\text { belonging to degradation level D, since the probability curve tends to } 100 \% \text { as age increases. }\end{array}$} \\
\hline
\end{tabular}




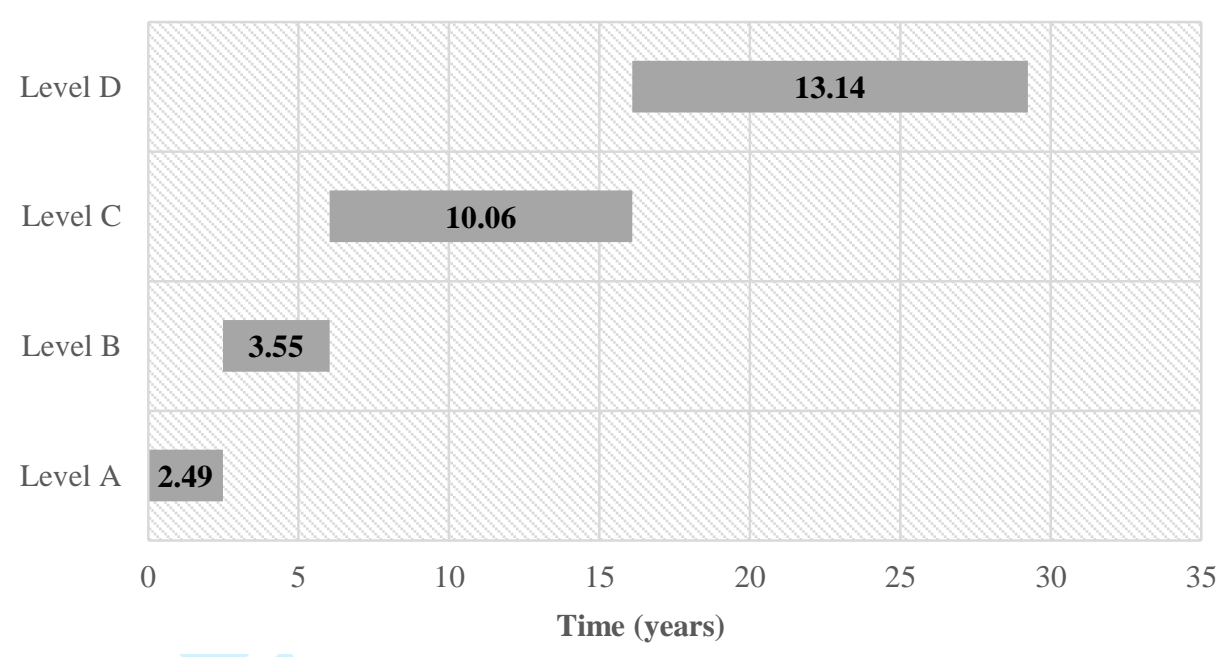

Figure 1 - Mean permanence time in each degradation state 


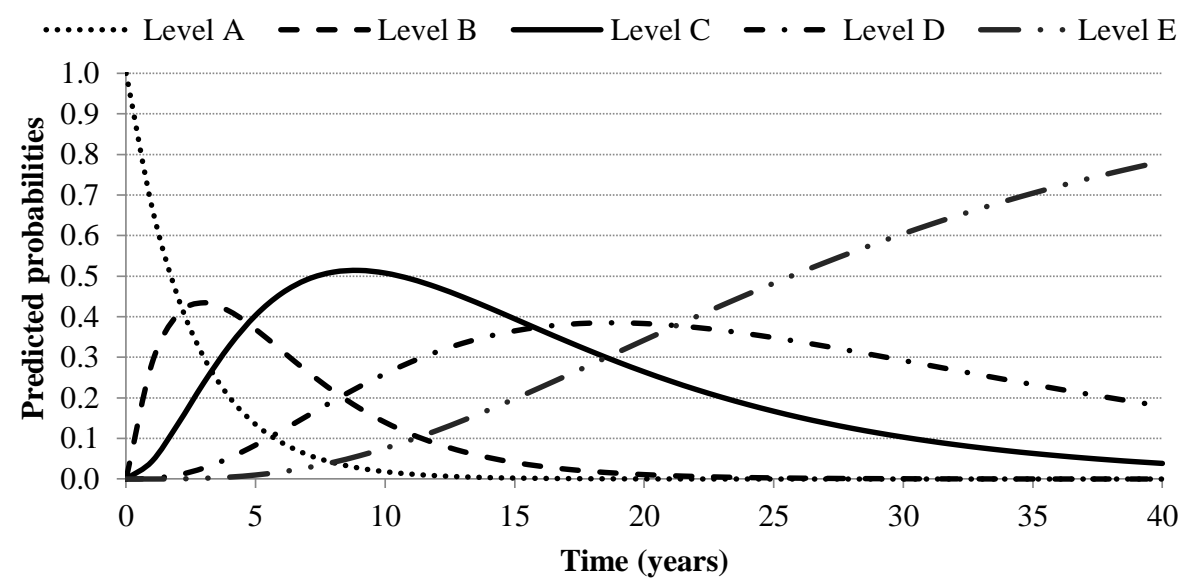

Figure 2 - Probabilistic distribution of the degradation condition according to the age of the case studies 


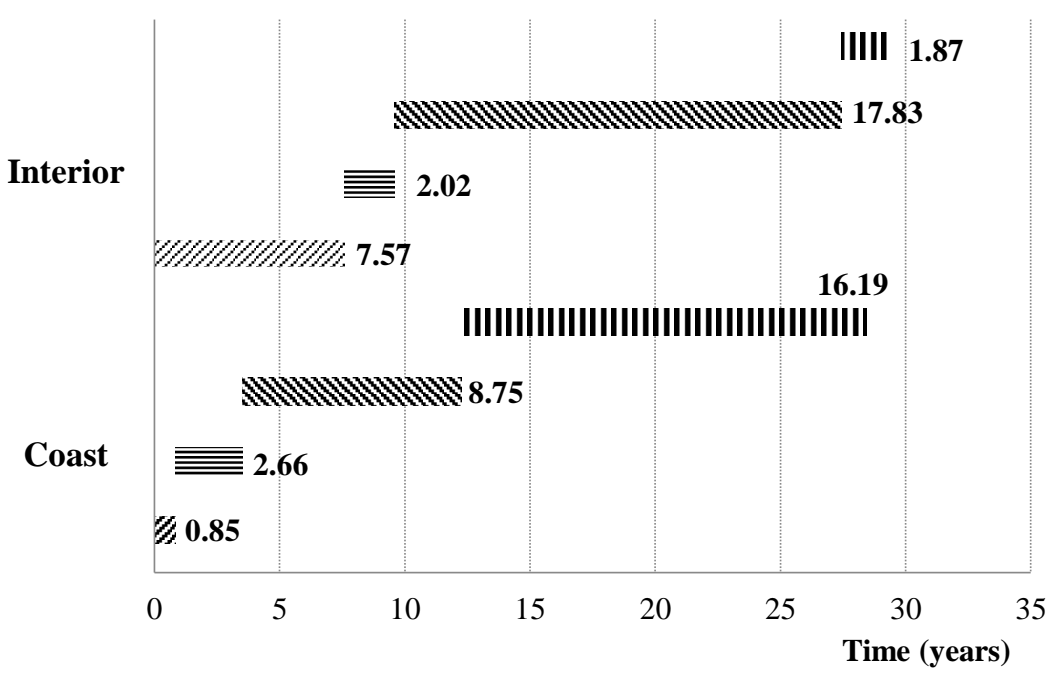

Figure 3 - Mean permanence time in each degradation state according to distance from the sea 

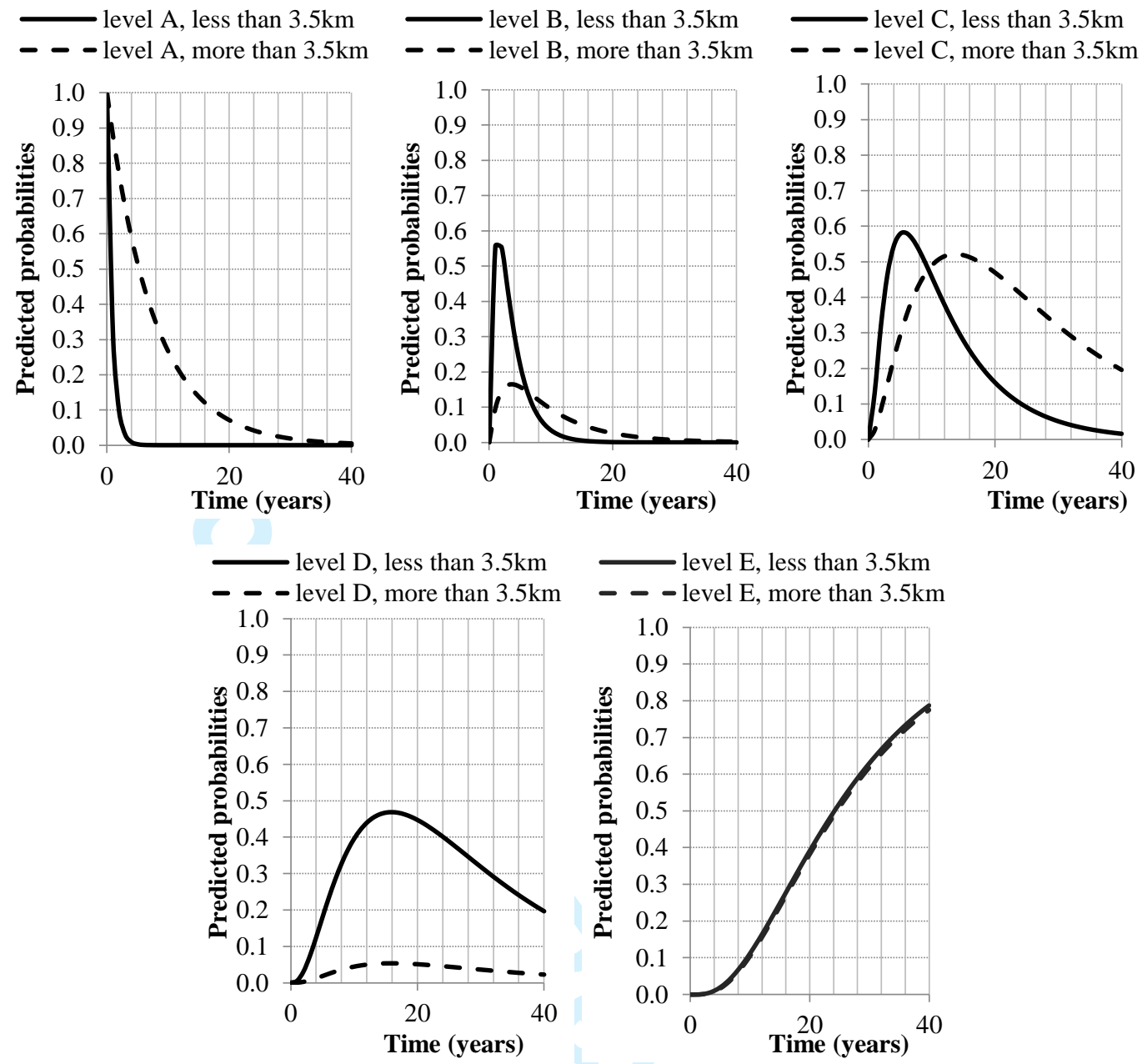

Figure 4 - Probabilistic distribution of the degradation condition according to distance from the sea 


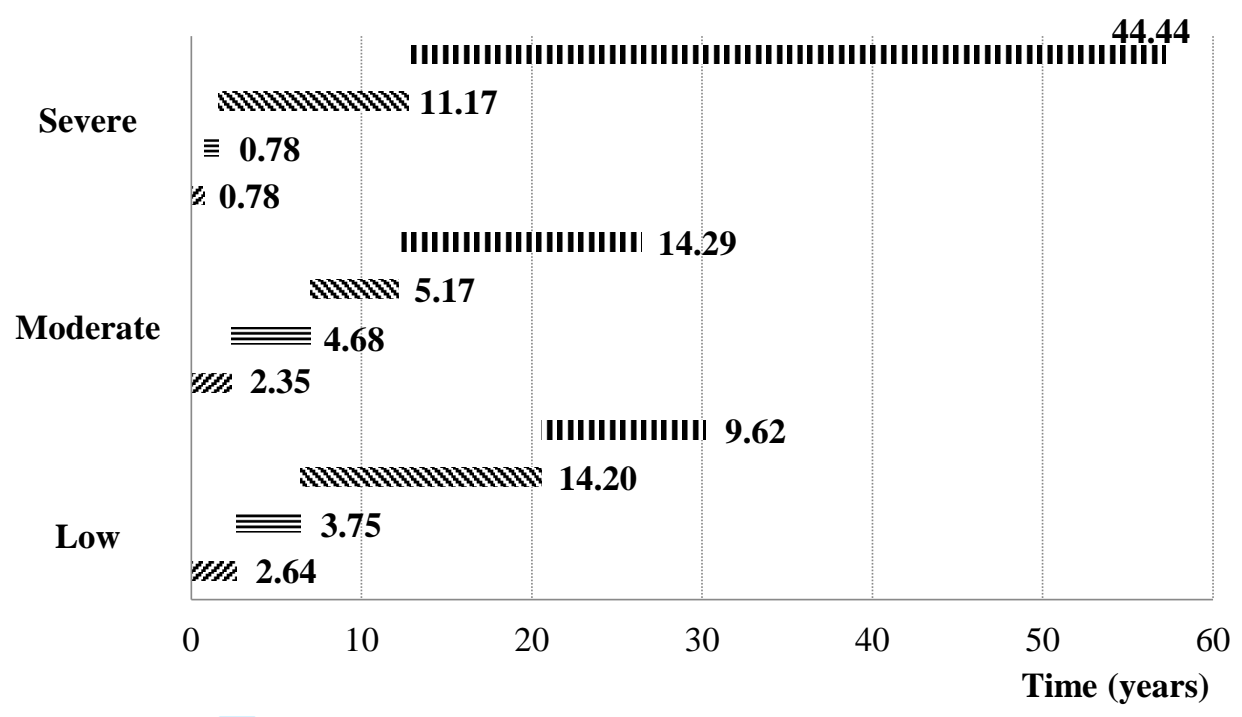

Figure 5 - Mean permanence time in each degradation state according to exposure to damp 

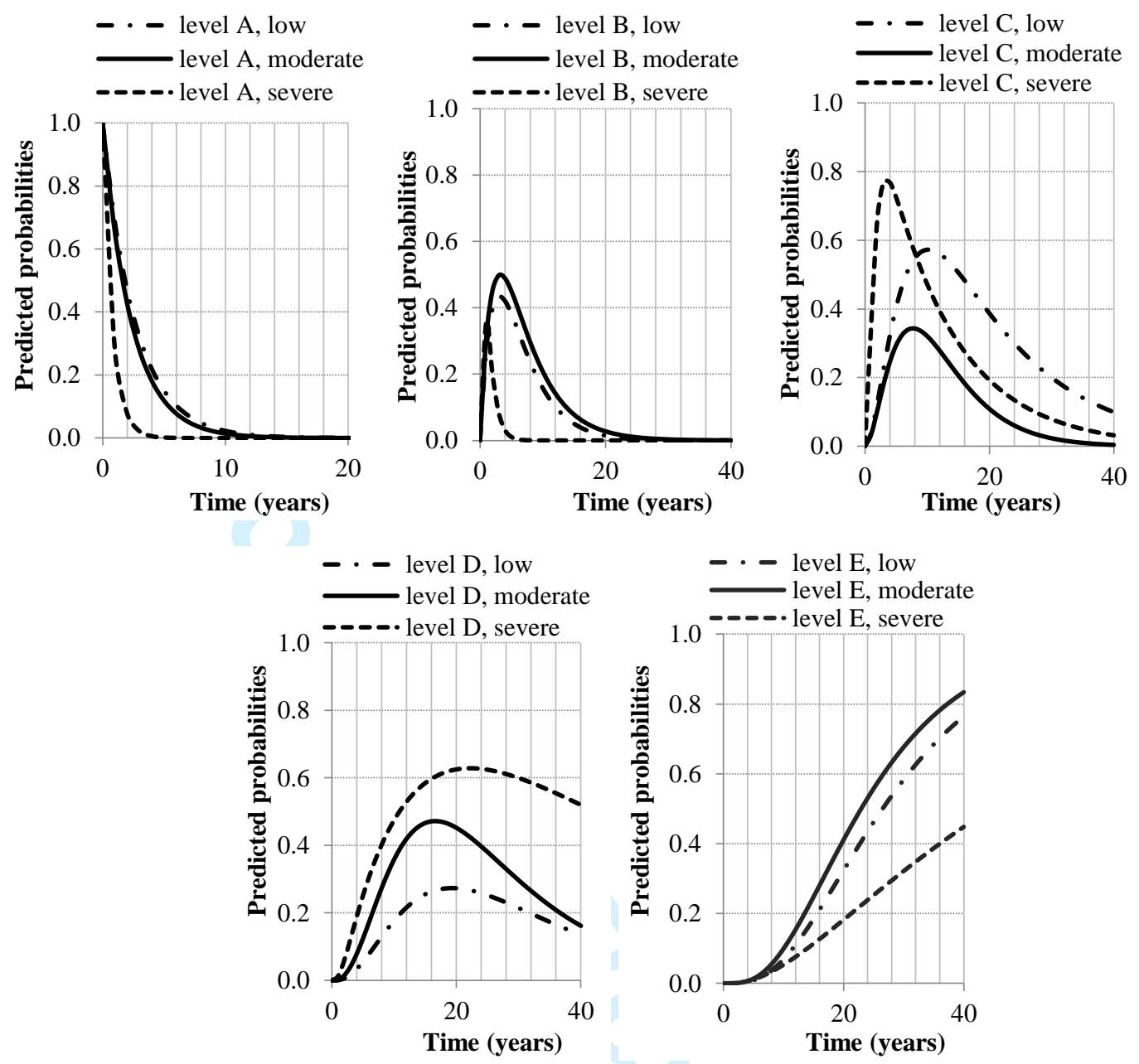

Figure 6 - Probabilistic distribution of the degradation condition according to exposure to damp 


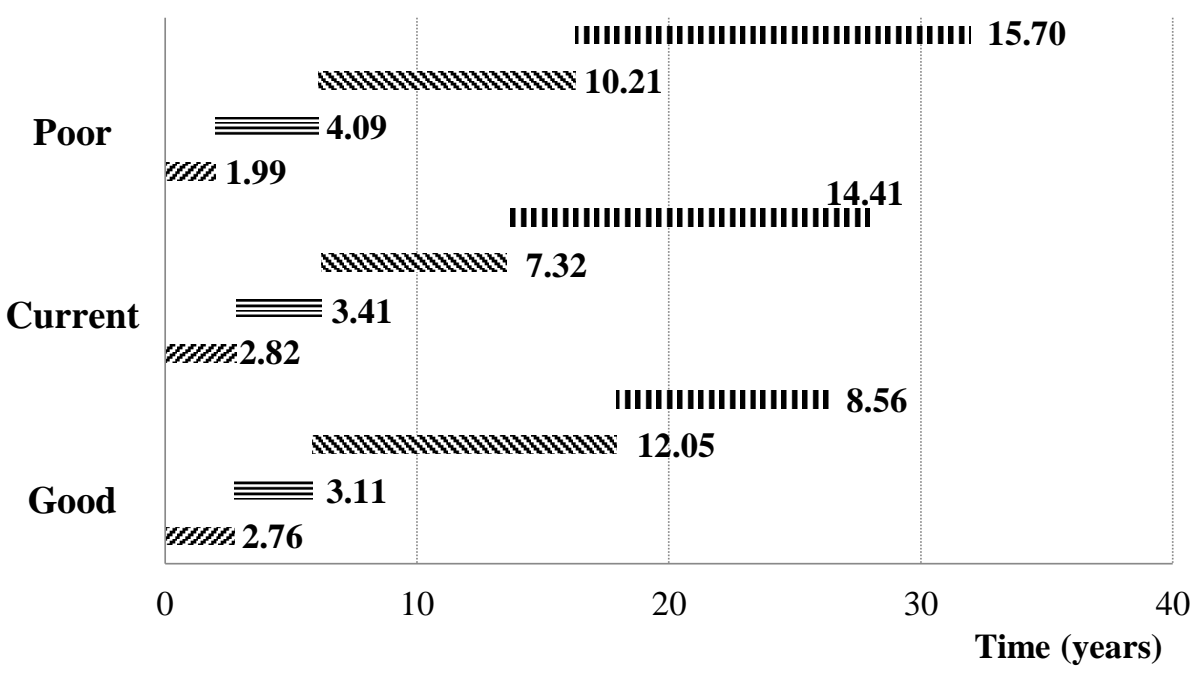

Figure 7 - Mean permanence time in each degradation state according to the protection level of the facades 

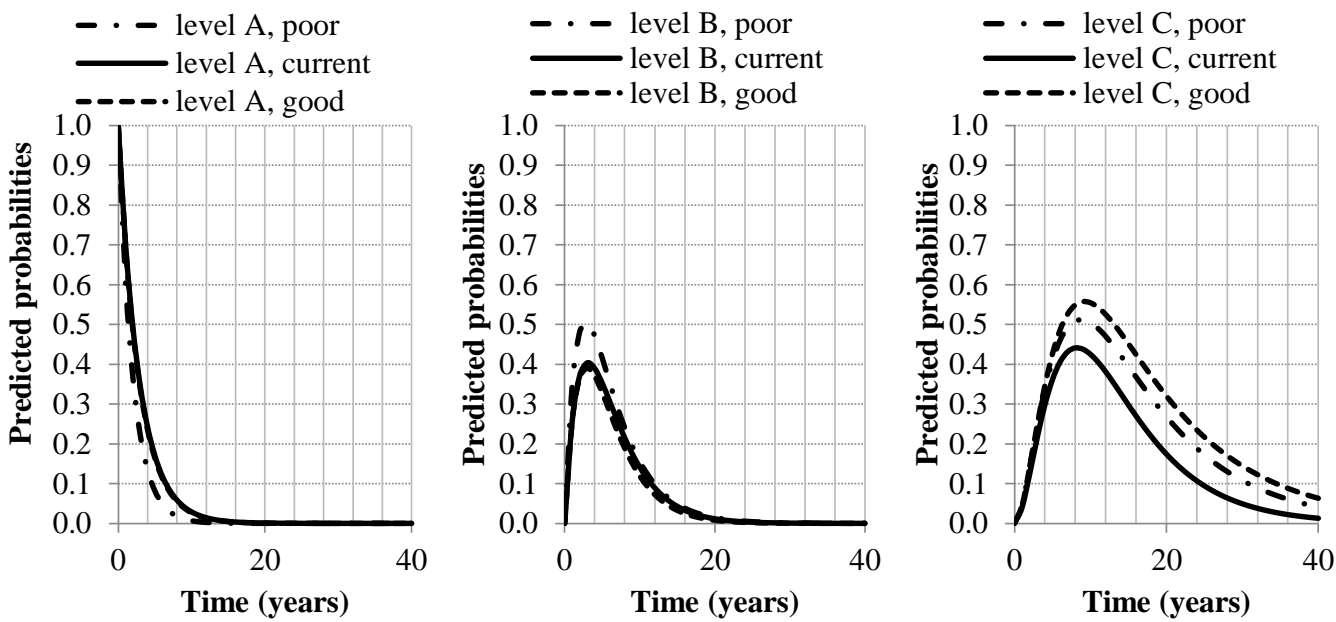

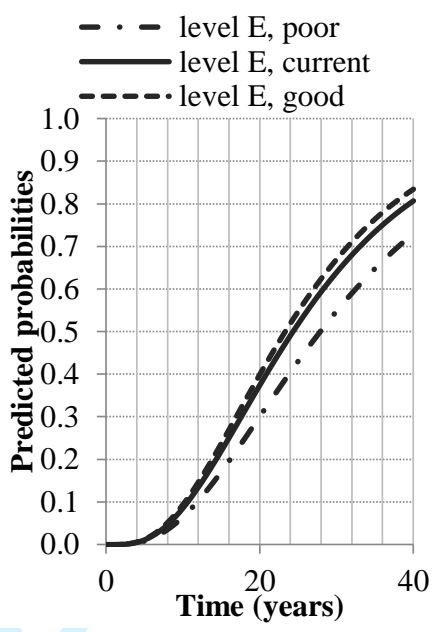

Figure 8 - Probabilistic distribution of the degradation condition according to the protection level of the facades 


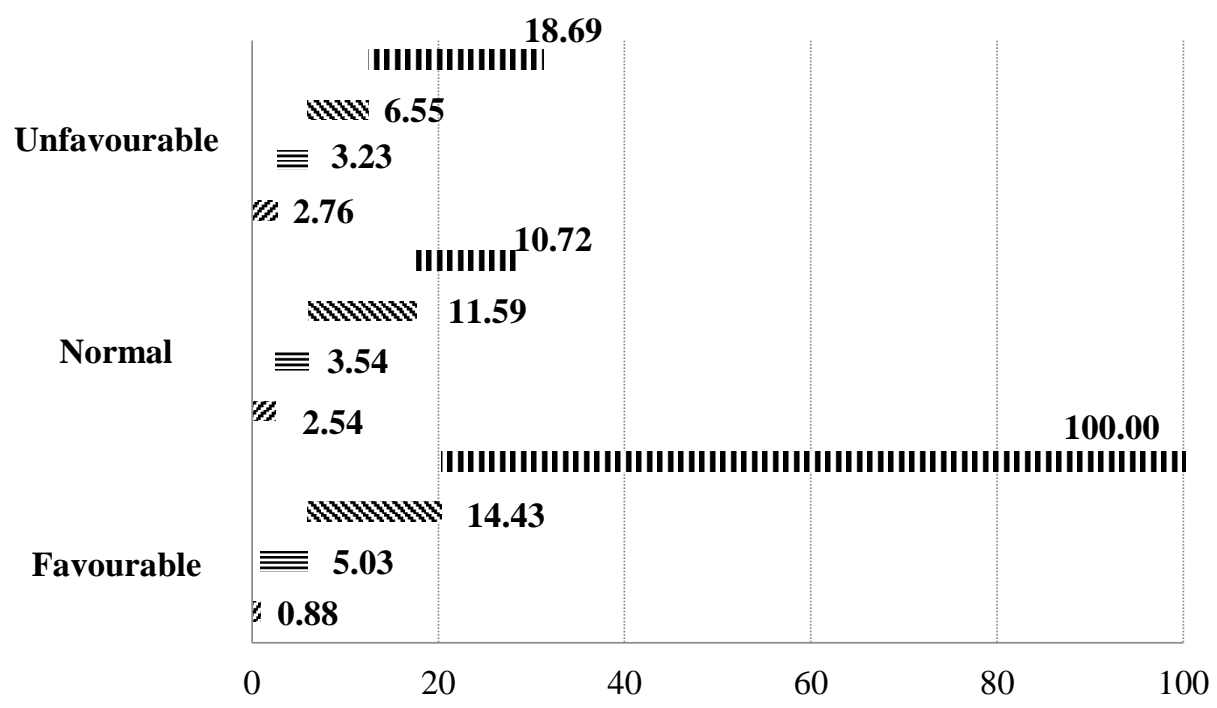

Time (years)

Figure 9 - Mean permanence time in each degradation state according to exposure to pollutants 

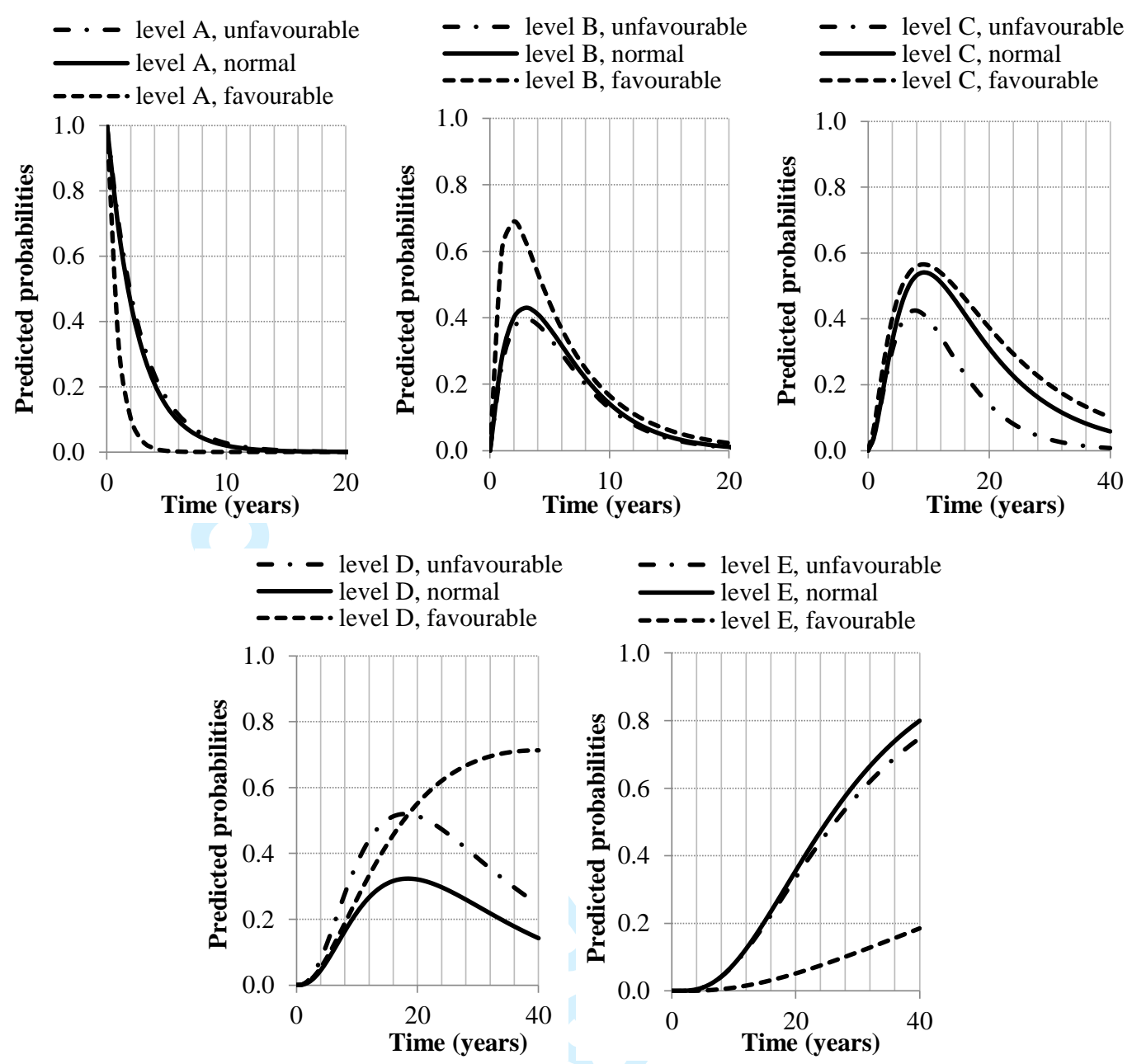

Figure 10 - Probabilistic distribution of the degradation condition according to exposure to pollutants 


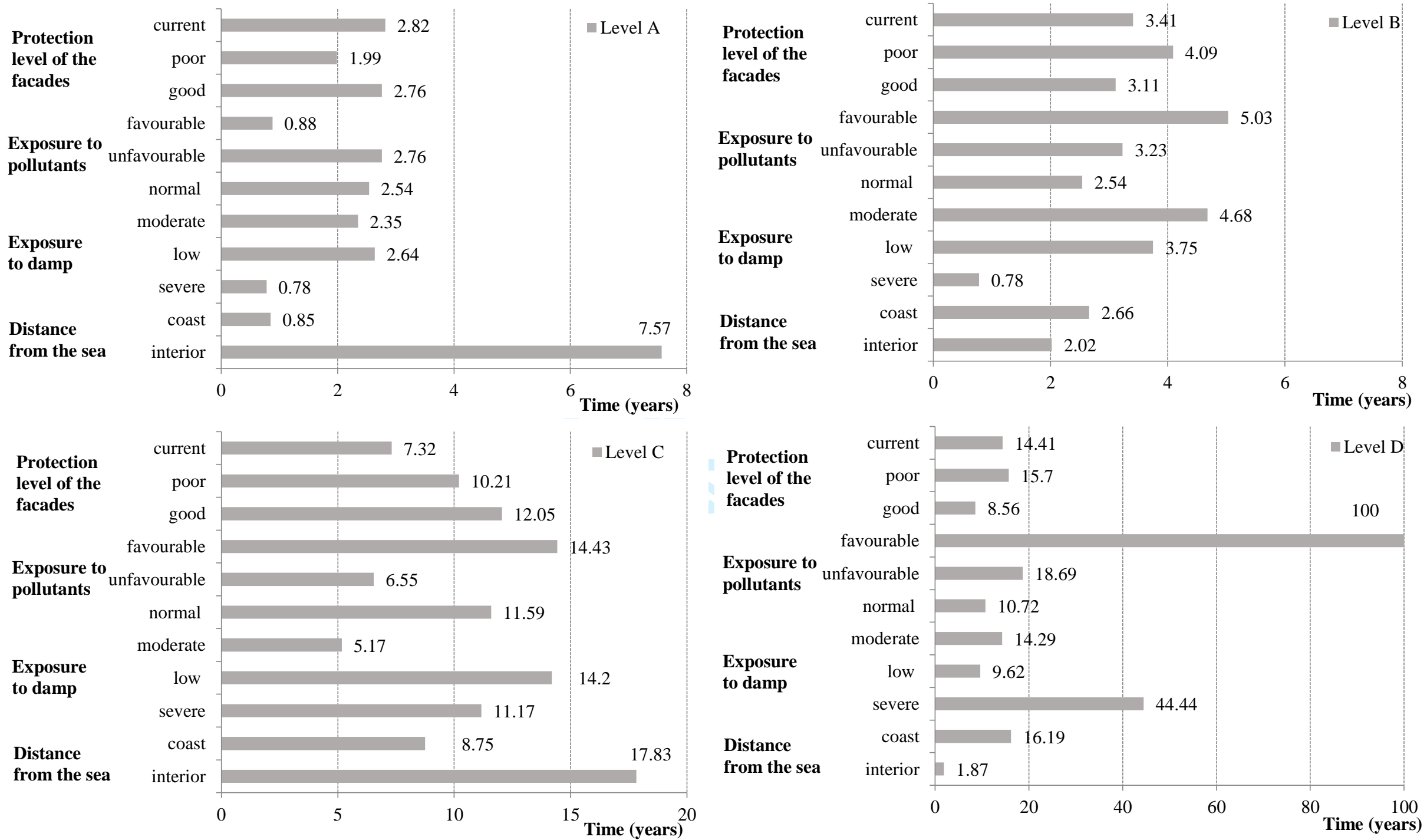

Figure 11 - Mean permanence time in each degradation state according to the environmental factors analysed 


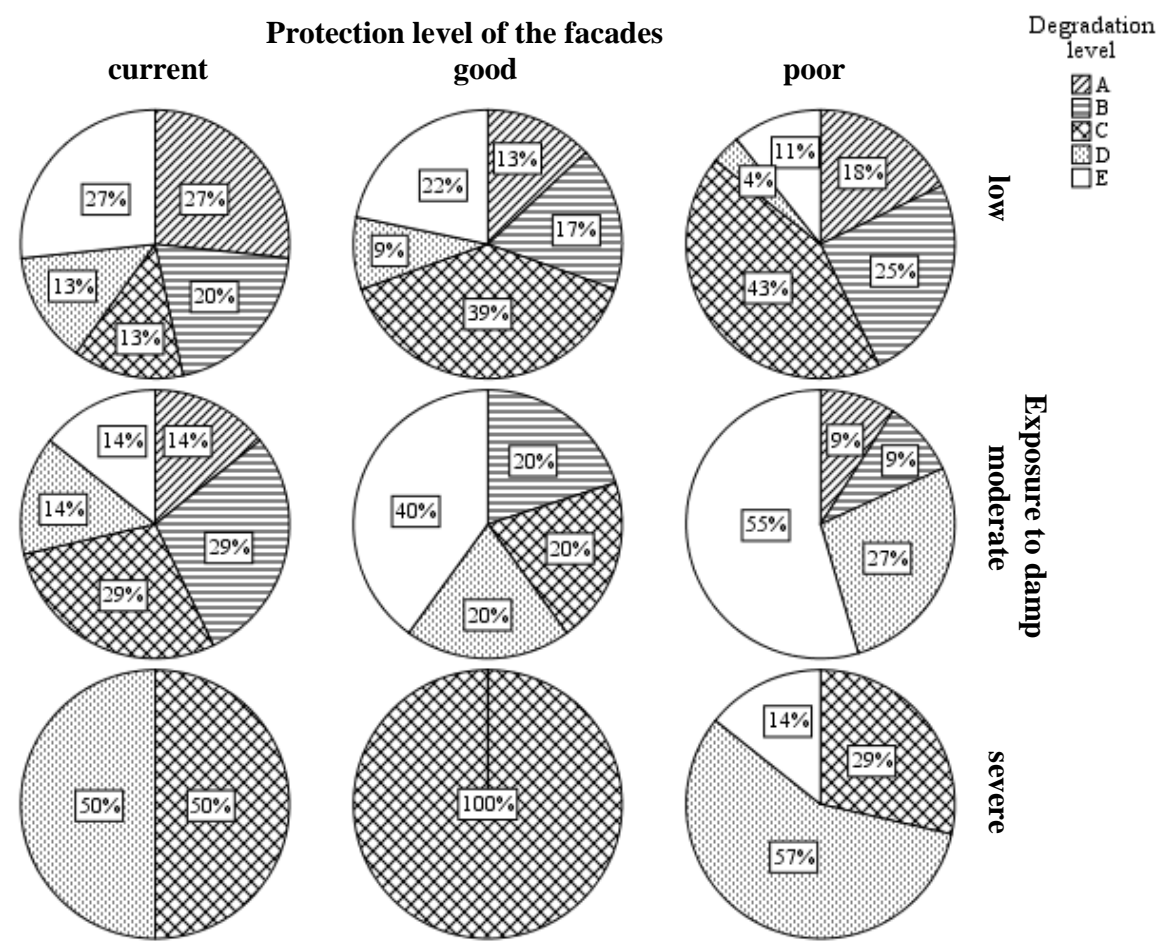

Figure 12 - Analysis of the different degradation levels according to the exposure to damp and the protection level of the facades 


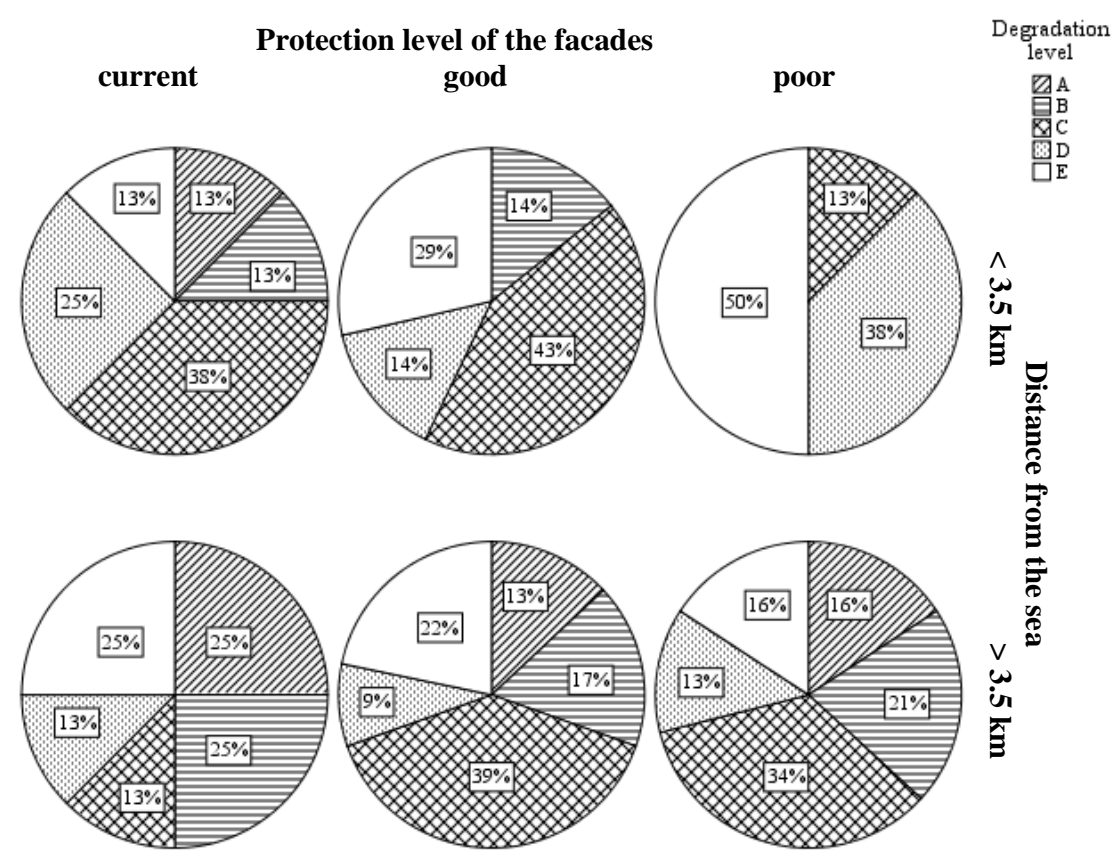

Figure 13 - Analysis of the different degradation levels according to the distance from the sea and the protection level of the facades 